\title{
Coherence of XUV Laser Sources
}

\author{
Sebastian Roling and Helmut Zacharias \\ Westfälische Wilhelms-Universität Münster
}

Germany

\section{Introduction}

This article reviews recent developments in research on coherence properties of femtosecond XUV and x-ray lasers. Coherence is one of the most conspicuous features of laser sources. It describes how an electromagnetic wave field is correlated at different times and at different points in space. Coherence is a property that enables stationary interferences and thereby enables holographic and diffractive or lens less imaging.

While a large number of different laser systems radiating in the infrared, visible and UV spectral range have been developed in the past five decades since the invention of the laser, the generation of intense and coherent XUV and $x$-ray radiation is still a formidable goal of basic research. Developed first in the mid and far infrared spectral region free electron lasers (FEL) provide a new means to generate widely tunable coherent radiation [Madey, 1971; Deacon et al., 1977; Oepts et al., 1995]. In the XUV and soft x-ray spectral region the present lack of appropriate mirrors prevent the construction of optical resonators, essential for a laser with well-defined mode properties. Therefore in this spectral region free-electron lasers have to rely on the principle of self-amplified spontaneous emission (SASE), where in a single path enough gain is accumulated to evolve a pulse from shot noise. These machines then provide pulsed XUV and x-ray radiation which shows only partial coherence (Kondratenko \& Saldin, 1980; Bonifacio et al., 1984; Murphy \& Pellegrini, 1985). A characterisation of the coherence properties of SASE FEL radiation is not only important for the understanding and improvement of the SASE process. With the concomitant development of coherent diffraction imaging it is also of great practical importance in a broad field of fundamental scientific applications, like holographic imaging of artificial magnetic structures or single pulse imaging of large biomolecules. The theoretical description of the coherence properties of SASE FEL has made tremendous progress in the recent years. In this chapter we will briefly review measurement methods and description of partially coherent optical fields. The considerations will be applied to characterize the spatial and temporal coherence of pulses from the SASE free electron laser in Hamburg (FLASH) and of high harmonic radiation.

\section{Basic definitions}

Coherence is a measure of the correlation properties of different electromagnetic wave fields. For a more general comprehension some basic definitions are reviewed following the books of Mandel and Wolf (Mandel \& Wolf, 1995) and Goodman (Goodman, 2000). The mutual correlation function $\Gamma$ describes the coherence of an electromagnetic field $E$ between two positions $\mathbf{r}_{1}$ and $\mathbf{r}_{2}$ at different times $t$ and $(t+\tau)$ : 


$$
\Gamma\left(\mathbf{r}_{1}, \mathbf{r}_{2}, \tau\right)=E\left(\mathbf{r}_{1}, t\right) E^{*}\left(\mathbf{r}_{2}, t+\tau\right)
$$

For a fixed distance $\left(\mathbf{r}_{\mathbf{i}}-\mathbf{r}_{\mathbf{j}}\right)$ this simplifies to the mutual time correlation function:

$$
\Gamma_{i j}\left(\mathbf{r}_{i}-\mathbf{r}_{j}, \tau\right)=E_{i}(t) E_{j}^{*}(t+\tau)
$$

The absolute value of the normalised correlation function $\gamma_{12}(\tau)$

$$
\left|\gamma_{12}(\tau)\right|=\Gamma_{12}(\tau) / \sqrt{\Gamma_{11}(0) \Gamma_{22}(0)} .
$$

can be measured as the visibility $\mathrm{V}$ of the interference fringes of two interfering partial beams. The visibility $\mathrm{V}$ is connected to the normalised correlation $\gamma_{12}(\tau)$ function via

$$
\mathrm{V}=\mathrm{\gamma}_{12}(\tau) \sqrt{\mathrm{I}_{1} \mathrm{I}_{2}} /\left(\mathrm{I}_{1}+\mathrm{I}_{2}\right)=\left(\mathrm{I}_{\max }-\mathrm{I}_{\min }\right) /\left(\mathrm{I}_{\max }+\mathrm{I}_{\min }\right),
$$

where $I_{1}$ and $I_{2}$ are the intensities of the interfering partial beams and $I_{\max }$ and $I_{\min }$ are the maximum and minimum intensities of the interference fringes, respectively. The coherence time $\tau_{\mathrm{c}}$ can be defined as the half width at half maximum [HWHM] of $\gamma_{12}(\tau)$. A more general definition for arbitrary functions is given by

$$
\tau_{c}=\int_{-\infty}^{\infty}\left|\gamma_{12}(\tau)\right|^{2} d \tau
$$

From the mutual correlation function $\Gamma$ the cross spectral density $W\left(\mathbf{r}_{1}, \mathbf{r}_{2}, \omega\right)$ is then defined as

$$
\mathrm{W}\left(\mathbf{r}_{1}, \mathbf{r}_{2}, \omega\right)=\int_{-\infty}^{\infty} \Gamma\left(\mathbf{r}_{1}, \mathbf{r}_{2}, \tau\right) \mathbf{e}^{\mathbf{i} \omega \tau} \mathrm{d} \tau
$$

From this expression one can readily recognize that it forms a Fourier pair with the mutual correlation function $\Gamma$. The cross spectral density constitutes a measure between the spectral amplitudes of any particular frequency component $\omega$ of the electromagnetic field at the spatial points $\mathbf{r}_{1}$ and $\mathbf{r}_{2}$. When the cross spectral density $W$ is treated for one point $\mathbf{r}_{\mathbf{1}}=\mathbf{r}_{\mathbf{2}}=\mathbf{r}$ it equals the power spectrum $S(\mathbf{r}, \omega)$ of the light

$$
\mathrm{S}(\mathbf{r}, \omega)=\mathrm{W}(\mathbf{r}, \mathbf{r}, \omega)
$$

Analogous to the mutual coherence function $\Gamma$ also the cross spectral density $W$ can be normalised

$$
\mu\left(\mathbf{r}_{1}, \mathbf{r}_{2}, \omega\right)=W\left(\mathbf{r}_{1}, \mathbf{r}_{2}, \omega\right) / \sqrt{S\left(\mathbf{r}_{1}, \omega\right)} \sqrt{S\left(\mathbf{r}_{2}, \omega\right)}
$$

The normalised cross spectral density $\mu\left(\mathbf{r}_{1}, \mathbf{r}_{2}, \omega\right)$ is usually referred to as the spectral degree of coherence. In analogy to the coherence time of the light pulse also the degree of transverse coherence $\zeta$ is defined as

$$
\zeta=\iint\left|\gamma\left(\mathbf{r}_{1}, \mathbf{r}_{2}\right)\right|^{2} \mathrm{I}\left(\mathbf{r}_{1}\right) \mathrm{I}\left(\mathbf{r}_{2}\right) \mathrm{d} \mathbf{r}_{1} \mathrm{~d} \mathbf{r}_{2} /\left[\int \mathrm{I}(\mathbf{r}) \mathrm{d} \mathbf{r}\right]^{2} .
$$

Here I(r) denotes the intensity distribution of the electromagnetic field. An experimentally easily accessible value for the transverse coherence is given by the (r.m.s) transverse 
coherence length $\xi_{x, y}$. The effective transverse coherence length at a distance $z$ from the source is $\Xi_{x, y}(z)=\xi_{x, y} \cdot \Sigma_{x, y}(z) / \sigma_{s x, y}$, where $\Sigma_{x, y}(z)$ denotes the r.m.s. beam size at the position $z$ and $\sigma_{\mathrm{s}}$ is the r.m.s source size. This article reviews recent theoretical and experimental results concerning the coherence time $t_{c}$, the degree of transverse coherence $\zeta$ and accordingly the transverse coherence length $\xi_{x, y}$ of free electron lasers and highharmonic generation light sources in the XUV and $x$-ray spectral regime.

\section{The SASE FEL process}

The generation of coherent radiation in a Self-Amplified Spontaneous Emission (SASE) process by a relativistic electron beam passing through a series of regularly spaced magnetic fields with opposite direction - a so-called undulator that is shown in Fig. 1a - was first proposed by Kondratenko and Saldin in 1980 (Kondratenko \& Saldin, 1980). In 1985 Murphy and Pellegrini (Murphy \& Pellegrini, 1985) pursued this idea of a single-pass amplifier starting from noise for soft $\mathrm{x}$-ray radiation. Since in the $\mathrm{x}$-ray spectral region optical cavities would feature significant losses present machines operate on a single-pass through an undulator. Therefore a SASE FEL consists of an electron accelerator and a sufficiently long undulator.
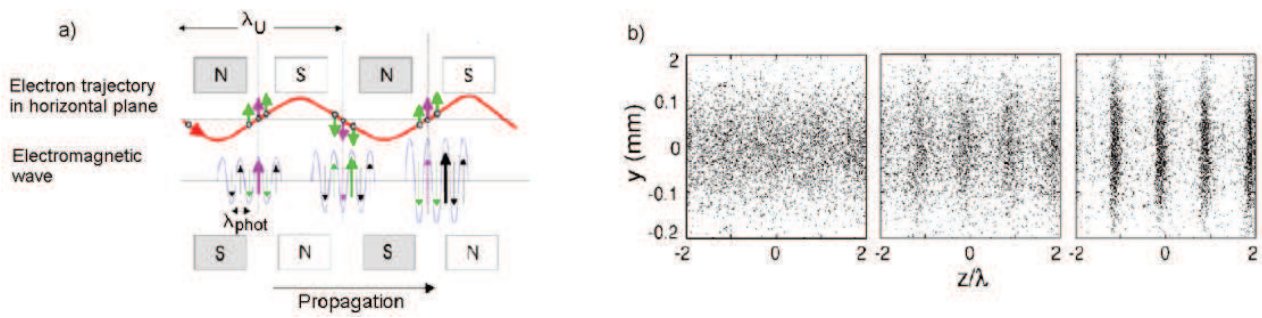

Fig. 1. (a) The electron orbit in a periodic undulator field (undulator period $\lambda_{\mathrm{U}}$ ). Three electrons and the field of an electromagnetic wave (wavelength $\lambda_{\text {phot }}$ ) are shown at three positions along the undulator. The phase slip of the electrons with respect to the light field by exactly one wavelength from one undulator period to the next is discernible. (b) The density modulation of the electron bunch as it develops along the undulator, at the beginning of the undulator, in the middle and at the exit. The pictures are taken from reference (Feldhaus, Arthur \& Hastings, 2005).

When the quality of the electron beam in terms of charge density, emittance and energy spread is high enough, the interaction between the electrons oscillating in the undulator magnetic field and the synchrotron radiation that is spontaneously emitted, leads to a density modulation of the electron bunch in the longitudinal direction, see Fig. 1b.

The period length of this modulation equals the wavelength of the undulator radiation (microbunching). Figure 1a shows a bunch of three electrons and the field of the electromagnetic wave at three positions along the undulator. An electron is accelerated or decelerated by the electric field of the radiation depending on its relative phase with respect to the light wave. This leads to a velocity modulation which then transforms into a longitudinal density modulation if the undulator is sufficiently long. The microbunched electrons will then radiate in phase with each other because of their longitudinal grouping 
being exactly one optical wavelength. Thereby, the microbunching is sharpened further which in turn enhances the intensity of the electromagnetic field. This effect is referred to as Self-Amplified Spontaneous Emission (SASE). The radiated intensity of such a FEL is therefore proportional to the square of the number of particles involved and presently reaches values of the order of $1 \mathrm{~mJ}$ per pulse (Feldhaus, Arthur \& Hastings, 2005; Ackermann et al., 2007; Emma et al., 2010). A list of some essential parameters of already operating and proposed SASE FEL can be found in Table I.

\begin{tabular}{|l|c|c|c|}
\hline & FLASH & LCLS & XFEL \\
\hline $\begin{array}{l}\text { Minimum Wavelength } \\
\text { (fundamental) [nm] }\end{array}$ & 4.45 & 0.12 & 0.1 \\
\hline $\begin{array}{l}\text { Maximum Photon energy } \\
\text { (fundamental) [eV] }\end{array}$ & 276 & 8270 & 12400 \\
\hline Pulse duration & $29 \mathrm{fs}$ at $24 \mathrm{~nm}$ & 500 to $<10 \mathrm{fs}$ & $100 \mathrm{fs}$ \\
\hline Coherence time & 6 fs at $24 \mathrm{~nm}$ & $<1 \mathrm{fs}$ & $0.22 \mathrm{fs}$ \\
\hline Pulse energy [mJ] & 0.3 & $1.3-3$ & 30 \\
\hline
\end{tabular}

Table I. Main parameters of FLASH (Mitzner, 2008; Mitzner, 2009; DESY Homepage, 2010), LCLS (McNeil, 2009; Emma et al., 2010), XFEL (Altarelli et al., 2006).

\section{Theoretical simulations}

Bonifacio et al. (Bonifacio et al., 1994a; 1994b) presented a systematic theoretical study of the temporal structure, the linewidth, noise, fluctuations and the spectrum of a SASE FEL. The latter is directly related to the temporal coherence (see equation 8). The results of the 1D time-dependent numerical model show that the temporal as well as the spectral structure consist of several spikes. Saldin et al. report a systematic approach for an analytical description of SASE FEL in the linear mode of amplification where SASE does not yet reach full saturation (Saldin et al., 2000b). In addition to the average radiated power and the spectral envelope also the angular distribution of the radiation intensity in the far field and the degree of transverse coherence was calculated. This general result was applied to the special case of an electron beam with a Gaussian profile and a Gaussian energy distribution. Applying the three dimensional time-dependent numerical code FAST (Saldin et al., 1999) an investigation of the amplification process in a SASE FEL was implemented. The fluctuations of the current density in the electron bunch are uncorrelated not only in time but also in space. In contrast to the 1D model this fact is taken into account within the 3D model. The results were compared with analytical results showing that in the high-gain linear limit there is good agreement between both approaches. Importantly, it was found that even after completion of the transverse mode selection process the degree of transverse coherence of the radiation from a SASE FEL significantly differs from the ideal situation of complete coherence. This is a consequence of the interdependence of the longitudinal and transverse coherence. The SASE FEL shows poor longitudinal coherence which develops only slowly with increasing undulator length and thus prevents a full transverse coherence. For sake of generality the undulator length $\mathrm{z}$ is replaced by the normalized undulator length

$$
\hat{z}=\Gamma \cdot z
$$


where $\Gamma=\left[\Pi j_{0} K_{1}^{2} / I_{A} \lambda_{U V}\right]^{1 / 3}$ denotes the gain parameter. Here $j_{0}$ is the beam current density, $\lambda_{\mathrm{U}}$ is the undulator period, $\mathrm{I}_{\mathrm{A}}=\mathrm{mc}^{3} / \mathrm{e} \approx 17 \mathrm{kA}$ is the Alfven current and $\omega=2 \pi \mathrm{c} / \lambda$ the frequency of the electromagnetic wave. The coupling factor $K_{n}$ is given as $\mathrm{K}_{\mathrm{n}}=\mathrm{K}(-1)^{(\mathrm{n}-1) / 2} \cdot\left[\mathrm{J}_{\mathrm{n}-1 / 2}(\mathrm{Q})-\mathrm{J}_{\mathrm{n}+1 / 2}(\mathrm{Q})\right]$, where $\mathrm{Q}=\mathrm{n} \mathrm{K}^{2} /\left[2\left(1+\mathrm{K}^{2}\right)\right]$. With the FEL efficiency parameter $\rho=c \gamma_{z}^{2} \Gamma / \omega$ the normalized coherence time is given as

$$
\hat{\tau}_{c}=\rho \omega \tau_{c} .
$$

The detuning $C$ is given as $C=k_{U}-\omega / 2 c \gamma_{z}^{2}$, where $k_{U}$ denotes the wave number of the undulator. The temporal structure of the radiation pulse at the undulator length $\hat{z}=15$ and a spectrum of the radiation from a SASE FEL calculated with the linear simulation code FAST are shown in Fig. 2. One can recognize that several temporal and spectral modes are present in the FEL radiation. Due to the connection between the frequency and time domain via a Fourier transformation a poor longitudinal coherence can readily be deduced from Fig. $2 \mathrm{~b}$. It had been found that the coherence time achieves its maximal value near the saturation point and then decreases drastically.
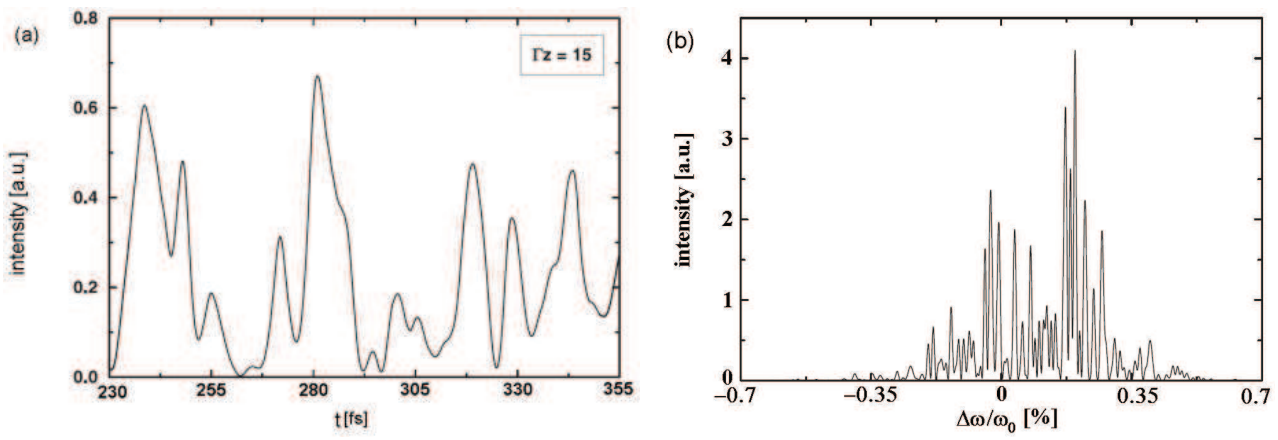

Fig. 2. (a) Temporal structure of the radiation pulse $(\lambda=13 \mathrm{~nm})$ at the undulator length $\hat{z}=$ 15. (b) Typical spectrum of the radiation from the FEL amplifier starting from shot noise at the undulator length $\hat{z}=15$. Both calculations had been performed with the linear simulation code FAST. After reference (Saldin et al., 2000b).

A simulation of the degree of transverse coherence of the radiation of a SASE FEL plotted versus the normalized undulator length is shown in Fig. 3. The transverse degree of coherence $\zeta$ was found to approach unity asymptotically as $(1-\zeta) \propto 1 / \mathrm{z}$ at $\Gamma \mathrm{z} \sim 15$ (Saldin et al., 2000b). At the beginning of the SASE process a large number $M$ of transverse radiation modes are excited when the electron beam enters the undulator. At that stage the fluctuations of the current density in the electron bunch are still uncorrelated not only in time but also spatially. Since these different modes have different spatial overlap with the electron beam the amplification they experience will be different. Therefore, the number of modes decreases upon further amplification. As the fundamental mode has the best overlap with the electron beam the total field amplitude is dominated by the fundamental mode, which contributes according to this simulation close to $99.9 \%$ to the total radiation power 
(Saldin et al., 2001). Transverse coherence establishes quickly at an early stage of amplification due to this transverse mode selection. One could mistakenly deduce that the latter effect proceeds further at higher $\mathrm{z}$ and the degree of transverse coherence should approach unity exponentially but not as $(1-\zeta) \propto 1 / \mathrm{z}$ as it is found in (Saldin et al., 2000b). However, at this point, one should take a closer look at the spiky temporal and spectral structure of the FEL pulses shown in Fig. 2. In the high-gain linear regime the radiation of the SASE FEL consists of a spatial fundamental mode, however, at many different frequencies. The transverse distributions of the radiation field of spatially fundamental modes are also slightly different for different frequencies. As a result of the spatial interference of these longitudinal modes full transverse coherence is not achieved during the SASE process. To conclude, the interdependence between longitudinal coherence and transverse coherence is accountable for the fact that full transverse coherence is not achieved even after completition the mode selection process.

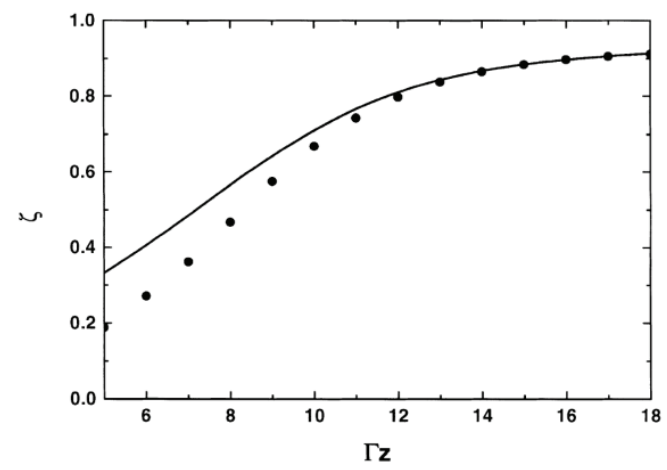

Fig. 3. The degree of transverse coherence $\zeta$ of the radiation from a FEL amplifier as a function of the undulator length. The solid curve represents analytical results and the circles are obtained with the linear simulation code FAST. Taken from reference (Saldin et al., 2000b).

Besides the fundamental radiation the microbunched electron pulse also supports gain at odd harmonics of the radiation, although with decreasing intensity. Saldin et al. evaluated the properties of third harmonic generation from SASE FEL by employing the timedependent FEL simulation code GINGER (Saldin et al., 2006). The maximal value of the coherence time depends on the saturation length and, therefore, on the number of cooperating electrons $N_{c}$. For the coherence time $\hat{\tau}_{c}$ of the fundamental harmonic they arrived at

$$
\left(\hat{\tau}_{c}\right)_{\max } \approx \sqrt{\Pi \ln \mathrm{N}_{\mathrm{c}} / 18} .
$$

The evolution of the longitudinal coherence for higher harmonics with increasing undulator length is shown in Fig. 4. It is obvious that the normalized coherence time $\hat{\tau}_{c}$ evolves in three different stages. Initially (up to $\hat{z}=7-8$, see Fig. 4) the coherence time increases linearly as it should occur for the spontaneous emission of the radiation from the undulator. When the process of nonlinear harmonic generation begins to dominate the spontaneous 
emission the coherence time drops sharply. Around $\hat{z}=10-11$ a plateau is obtained where the ratio of the coherence time of the $n$th harmonic to that of the first harmonic scales with $1 / \sqrt{n}$. The structure of the third harmonic radiation indicates a similar spiky characteristic both in time as well as in the spectral distribution (Saldin et al., 2006), as shown for the fundamental in Fig. 2. It was found that a sharpening of the peaks in the intensity distribution leads to the decrease of the coherence time for the higher harmonics. When the amplification process enters the nonlinear regime at a normalized undulator length of $\hat{z} \geq 11$, the relative sharpening of the intensity peaks of higher harmonics becomes stronger, and the coherence time decreases even further. In fact, the coherence time at the saturation point of $\hat{z}=13$ develops for higher harmonics approximately inversely proportional to the harmonic order $n$, as is shown in Fig.4.

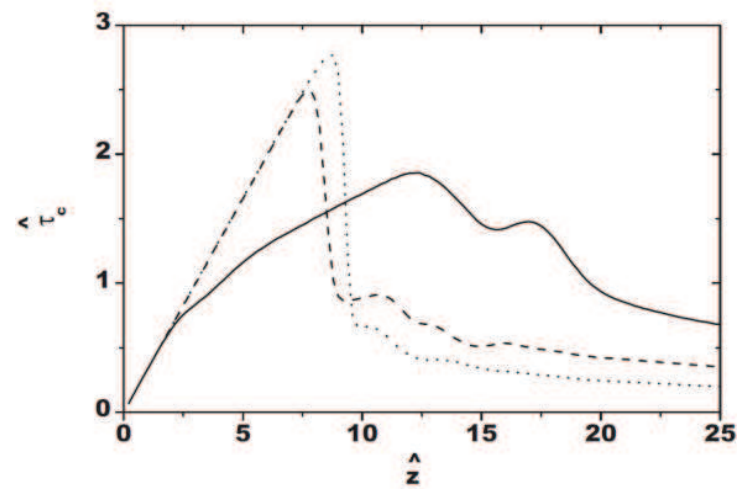

Fig. 4. Normalized coherence time of a SASE FEL as a function of normalized undulator length. Solid, dashed, and dotted lines correspond to the fundamental, 3rd, and 5th harmonic, respectively. Taken from reference (Saldin et al., 2006).

\section{Experimental results}

The experimental investigation of the spatial and temporal coherence properties of SASE FELs is of crucial importance, both from a fundamental point of view to assess different theoretical models, as well as for envisioned applications such as coherent imaging and holography in the x-ray spectral regime (Eisebitt et al., 2004; Chapman et al., 2006).

\subsection{Transverse coherence}

The first measurements of the transverse coherence of a SASE FEL operating in the VUV spectral regime have been performed by Ischebeck and co-workers in a Youngs double slit experiment (Ischebeck et al., 2003). Double slits with different separations, crossed slits and circular apertures were used to study the transverse coherence of the VUV light of the TESLA Test Facility, the predecessor of FLASH at DESY. The set-up is shown schematically in Fig. 5. It basically consisted of a Ce:YAG scintillator to convert the VUV light with wavelengths between $\lambda=80 \mathrm{~nm}$ and $\lambda=120 \mathrm{~nm}$ into visible light and a camera. The spatial distribution of the FEL radiation as well as the interference patterns from the different insertable apertures were observed. With a grating spectrometer equipped with an 
intensified CCD camera the spectral distribution of the light has been measured, although consecutively to the coherence measurements. To measure the transverse coherence properties double slits (width $\mathrm{d}=100 \mu \mathrm{m}$ and $\mathrm{d}=200 \mu \mathrm{m}$ ) with different slit separations between $0.5 \mathrm{~mm}$ and $3 \mathrm{~mm}$ were inserted at a distance of $11,84 \mathrm{~m}$ downstream from the undulator exit.

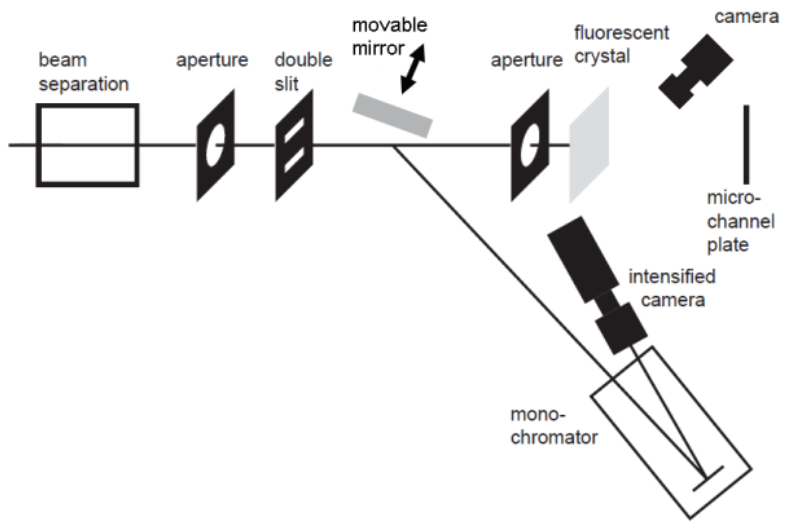

Fig. 5. The experimental set-up used for the first measurements of the transverse coherence at the TESLA Test Facility, after (Ischebeck et al., 2003).

Figure 6 shows the measured visibility (eqn. 4, circles) decreasing from $\mathrm{V}=0.83$ for $0.5 \mathrm{~mm}$ slit separation to $\mathrm{V}=0.23$ for $3.0 \mathrm{~mm}$ slit separation. The measured beam profile (asterisks) with a Gaussian fit (solid line) and a simulated beam profile taken from Saldin et al. (Saldin, Schneidmiller \& Yurkov, 1999) are also shown. The experimental results yield a high degree of transverse coherence of up to $\mathrm{V}=0.83$ in the center of the beam as it is expected from FEL theory presented above. The spatial coherence then decreases rapidly with increasing radial distance from the center of the beam.

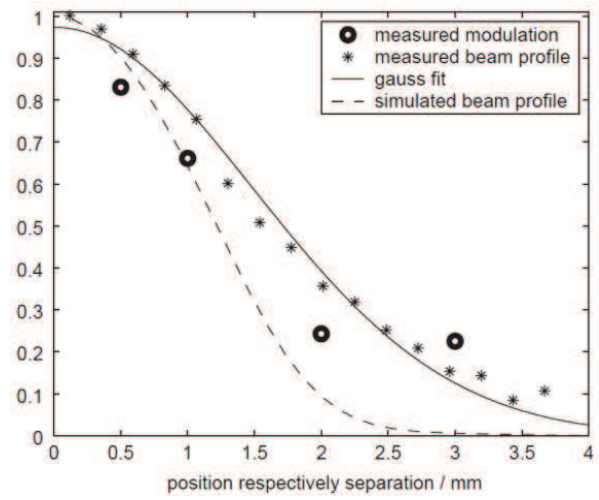

Fig. 6. Visibility in the center of the interference pattern as a function of the slit separation. For comparison, the beam profile measured with a MCP detector as a function of the position is shown. Taken from reference (Ischebeck et al., 2003). 
Singer et al. used Youngs double slit to measure the transverse coherence properties of FLASH operated at $\lambda=13.7 \mathrm{~nm}$ (Singer et al., 2008). The experimental results are compared to results from a phenomenological modeling in terms of the Gaussian-Shell model (GSM). For the measurements a Young's double slit experiment with slit separations of 150, 300 and $600 \mu \mathrm{m}$ was used. The double slit was positioned $20 \mathrm{~m}$ downstream from the undulator. Figure 7 shows the experimentally obtained absolute value of the complex degree of coherence with a Lorentzian fit yielding a transverse coherence length of $\xi_{x}=300 \pm 15 \mu \mathrm{m}$ for the horizontal direction and $\xi_{\mathrm{y}}=250 \pm 13 \mu \mathrm{m}$ for the vertical direction. The degree of coherence $|\gamma|$ decreases faster with the beam radius then the intensity. It is clear that the radiation field is not fully coherent, but rather partially coherent as it is expected from the measurements shown in reference (Ischebeck et al., 2003).

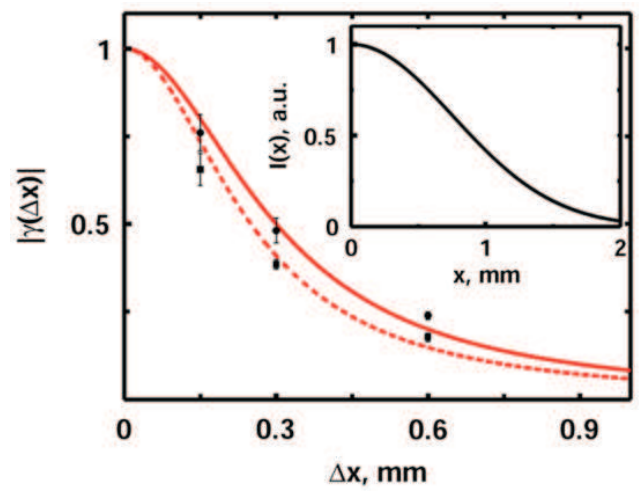

Fig. 7. Absolute value of the complex degree of coherence $|\gamma|$ at $\lambda=13.7 \mathrm{~nm}$ as a function of slit separation in the horizontal (circles) and vertical (squares) directions as a result of the fit to experimental data. A Lorentzian fit to the data of $|\gamma(\Delta x)|$ is shown by solid (horizontal direction) and dashed (vertical) lines. The intensity distribution is shown in the inset. Taken from reference (Singer et al., 2008).

For an analysis of the coherence properties of FLASH the experimental results are compared to the results from a general theoretical approach which is based on a decomposition of statistical fields into a sum of independently propagating transverse modes. This method is known as the Gaussian-Schell model (GSM). Thereby the crossspectral density $W\left(\mathbf{r}_{1}, \mathbf{r}_{2}\right)$ (see eqn. 6) is represented as a sum of independent coherent modes $\Psi\left(\mathbf{r}_{1}, \mathbf{r}_{2}\right)$

$$
\mathrm{W}\left(\mathbf{r}_{1}, \mathbf{r}_{2}\right)=\sum_{\mathrm{j}=0} \beta_{\mathrm{j}} \Psi_{\mathrm{j}}^{*}\left(\mathbf{r}_{1}\right) \Psi_{\mathrm{j}}\left(\mathbf{r}_{2}\right)
$$

Here $\Psi_{j}$ and $\beta_{\mathrm{j}}$ are the amplitudes and coefficients, respectively. To evaluate the coherence properties of the FEL the real FEL source was substituted by an equivalent model planar source described by a Gaussian Schell-model (GSM) cross spectral density

$$
\mathrm{W}\left(\mathbf{r}_{1}, \mathbf{r}_{2},\right)=\mathrm{A}_{0}^{2} \cdot \exp \left[-\frac{\left(\mathbf{r}_{1}^{2}+\mathrm{r}_{2}^{2}\right)}{4 \sigma_{\mathrm{I}}^{2}}-\frac{\left(\mathbf{r}_{1}+\mathbf{r}_{2}\right)^{2}}{2 \sigma_{\mu}^{2}}\right]
$$


Here $\sigma_{\mathrm{I}}$ and $\sigma \mu$ are the standard deviations of the spectral density and spectral degree of coherence at the source plane, respectively. The coordinates $\mathbf{r}_{1}$ and $\mathbf{r}_{2}$ are located in the plane of the source and $\mathrm{A}_{0}^{2}$ is the normalization constant. The analysis shows that six transverse modes are contributing to the total radiation field of FLASH. From Fig. 8a one can easily see that the contribution of the modes falls exponentially with the mode number $\mathrm{j}$. Thereby the contribution of the first mode is about $59 \%$ and the contribution of the second mode is $24 \%$ of the total radiation power. The contribution of the sixth mode is more than two orders of magnitude smaller. From Fig. $8 \mathrm{~b}$ a transverse coherence length of $\xi_{\mathrm{x}}=715 \mu \mathrm{m}$ can be obtained. Thus the experimentally measured values were considerably lower than those calculated in terms of the GSM. The apparent source size corresponding to the measured values for the coherence length was calculated making use of the GSM. The resulting value of $\sigma_{\mathrm{I}}=180 \mu \mathrm{m}$ was in good agreement with the source size observed in wave front measurements (Kuhlmann et al., 2006), but 2.5 times larger than considered in the theoretical modeling.
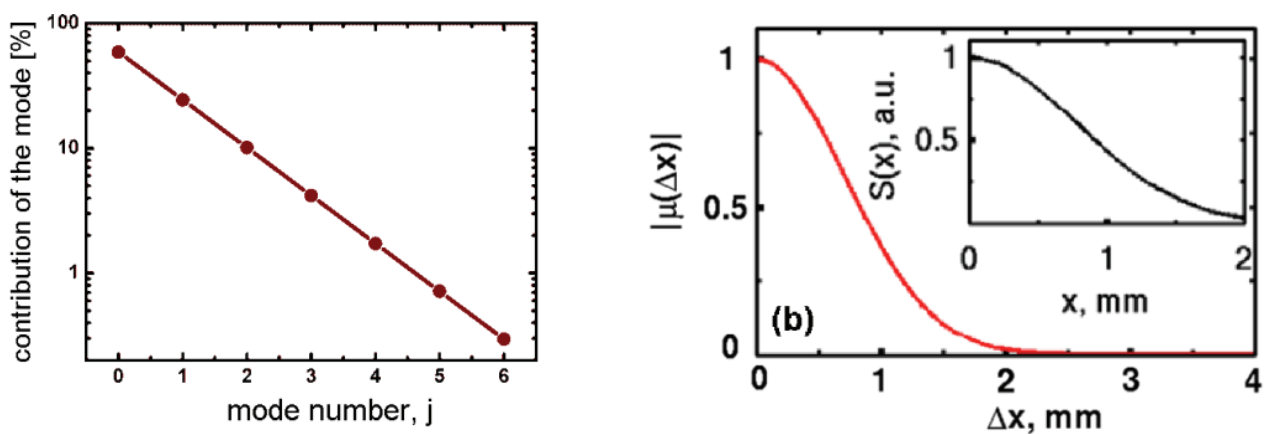

Fig. 8. (a) Contribution of individual modes in the GSM to the cross spectral density. (b) Absolute value of the spectral degree of coherence taken along a line through the middle of the beam profile. In the inset the spectral density $S(x)$ is shown. Calculations for the FEL operating in saturation are performed in the frame of a Gaussian-Schell model $20 \mathrm{~m}$ downstream from the source at a wavelength $\lambda=13.7 \mathrm{~nm}$; after reference (Singer et al., 2008).

Recently Vartanyants \& Singer employed the GSM to evaluate the transverse coherence properties of the proposed (Altarelli et al., 2006) SASE 1 undulator of the European XFEL, scheduled to begin operation in 2014. Simulations were made for a GSM source with an rms source size $\sigma_{\mathrm{s}}=27.9 \mu \mathrm{m}$ and a transverse coherence length $\xi_{\mathrm{s}}=48.3 \mu \mathrm{m}$ at the source for a wavelength of $\lambda=0.1 \mathrm{~nm}$ (corresponding to $\mathrm{h} \cdot v=12 \mathrm{keV}$ ), taken from the XFEL technical design report (Altarelli et al., 2006). Figure 9 shows the evolution of the beam size $\Sigma(\mathrm{z})$ and the transverse coherence length $\Xi(\mathrm{z})$ with the distance of propagation $\mathrm{z}$. At a distance $\mathrm{z}=$ $500 \mathrm{~m}$ from the source a transverse coherence length of $\Xi(\mathrm{z})=348 \mu \mathrm{m}$ and a beam size of $\Sigma(\mathrm{z})=214 \mu \mathrm{m}$ is obtained for the XFEL. Thus the coherence decreases less rapid than the spatial intensity of the beam. At present this prediction differs significantly from the experimental results for both TTF and FLASH. Distinct from a synchrotron source the coherence properties of the radiation field from the XFEL is of the same order of magnitude both for the vertical and the horizontal direction. 


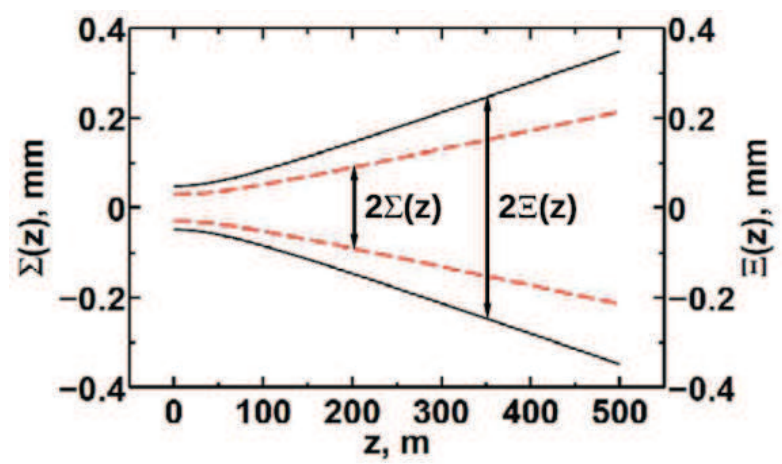

Fig. 9. Beam size $\Sigma(z)$ (dashed line) and the transverse coherence length $\Xi(z)$ (solid line) at different distances $z$ from the SASE 1 undulator of the European XFEL, from (Vartanyants \& Singer, 2010).

Earlier, results of numerical time dependent simulations for the coherence properties of LCLS XFEL at SLAC in Stanford have been reported (Reiche, 2006). For a wavelength of $\lambda=$ $0.15 \mathrm{~nm}$ (corresponding to $\mathrm{h} \cdot \mathrm{v}=8.27 \mathrm{keV}$ ) the effective coherence area within which the field amplitude and phase have a significant correlation to each other amounts to $0.32 \mathrm{~mm}^{2}$. That is about five times larger than the spot size with a value of $0.044 \mathrm{~mm}^{2}$ when evaluated at the first experimental location $115 \mathrm{~m}$ downstream from the undulator.

\subsection{Temporal coherence}

For an experimental measurement of the temporal coherence ideally time-delayed amplitude replicas of the FEL pulses should be brought to interference. However, the lack of amplitude splitting optical elements in the x-ray regime permits only the use of wavefront splitting mirrors in grazing incidence. These elements can then, however, be applied in a broad spectral region. Such a beam splitter and delay unit is shown schematically in Fig. 10(a).

(a)

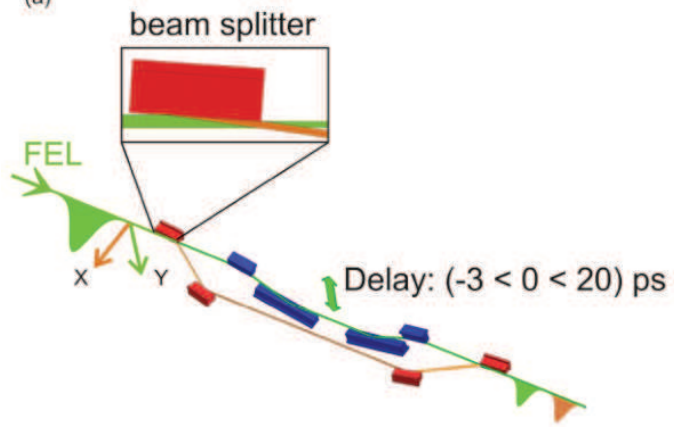

(b)

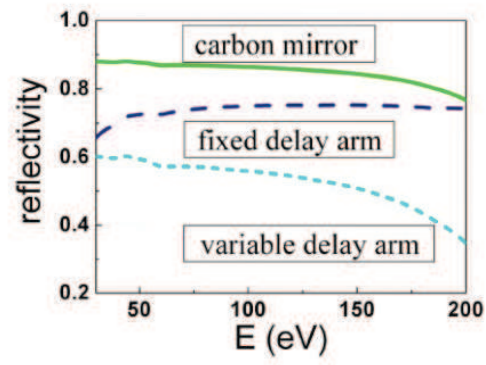

Fig. 10. (a) Schematic drawing of the layout of the autocorrelator. Grazing angles of $3^{\circ}$ and $6^{\circ}$ for the fixed and variable delay arms, respectively, are employed to ensure a high reflectivity of the soft $\mathrm{x}$-ray radiation. (b) Calculated reflectivity for amorphous carbon coated silicon mirrors for $\mathrm{h} v=30$ to $200 \mathrm{eV}$. The full green line shows the reflectivity of a single mirror for a grazing angle of $6^{\circ}$. 
Based on geometrical wave front beam splitting by a sharp mirror edge and grazing incident angles the autocorrelator covers the fundamental energy range of FLASH $(20-200 \mathrm{eV})$ with an efficiency of better than 50\%, see Fig. 10 (b). Grazing angles of $3^{\circ}$ and $6^{\circ}$ for the fixed and variable delay arms, respectively, are employed to ensure a high reflectivity of the soft $x$-ray radiation. Looking in the propagation direction the beam splitter with a sharp edge reflects the left part of the incoming FEL pulse horizontally into a fixed beam path. The other part of the beam passes this beam splitting mirror unaffected and is then reflected vertically by the second mirror into a variable delay line. A variable time delay between -5 ps and +20 ps with respect to the fixed beam path can be achieved with a nominal step size of 40 as. The seventh and eighth mirror reflect the partial beams into their original direction. Alternatively, small angles can be introduced to achieve and vary a spatial overlap of the partial beams. Mitzner et al. investigated the temporal coherence properties of soft $x$-ray pulses at FLASH at $\lambda=23.9 \mathrm{~nm}$ by interfering two time-delayed partial beams directly on a CCD camera (Mitzner et al., 2008). Fig. 11 shows two interferograms at zero and 50 fs delay, respectively. The overlap of the two partial beams is $\Delta x \approx 1.2 \mathrm{~mm}$ which corresponds $\sim 44 \%$ of the beam diameter in this case where an $1 \mathrm{~mm}$ aperture is set $65 \mathrm{~m}$ in front of the detector near the center of the beam profile. In these particular cases the contrast of the interference fringes yields via equation (4) a visibility of $\mathrm{V}=0.82$ and $\mathrm{V}=0.07$, respectively.
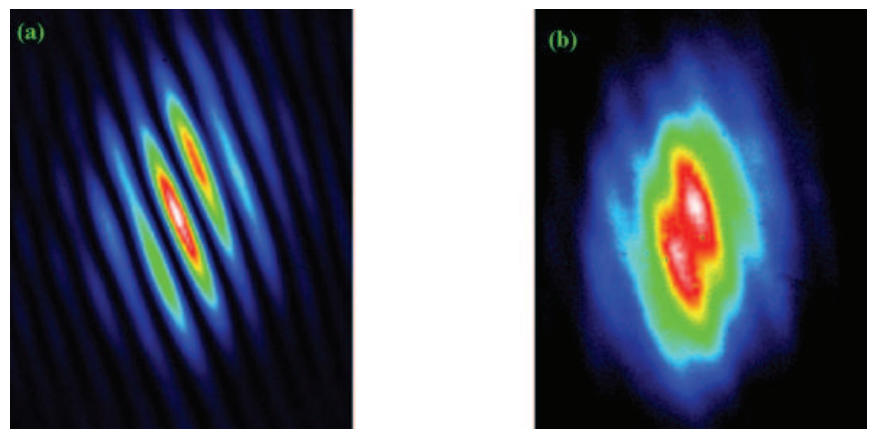

Fig. 11. Single exposure interference fringes at $\lambda=24 \mathrm{~nm}(\mathrm{a})$ at zero and (b) at $55 \mathrm{fs}$ delay between both partial beams. The crossing angle of the partial beams is $\alpha=60 \mu \mathrm{rad}$.

Scanning now the delay between the two pulses and calculating at each time step the visibility of the interference fringes (applying Equation 4) the temporal coherence properties of FLASH pulses are investigated. Figure 12 shows the time delay dependence of the average visibility observed for two different wavelengths, $\lambda=23.9 \mathrm{~nm}$ and $\lambda=8 \mathrm{~nm}$. Each data point (red dots) is the average of the visibility of ten single exposure interference pictures. In Fig. 12(a) the (averaged) visibility of $\mathrm{V}=0.63$ at zero time delay rapidly decreases as the time delay is increased. The central maximum of the correlation can be described by a Gaussian function (green line) with a width of $12 \mathrm{fs}$ (FWHM). Then a coherence time corresponding to half of the full width of $\tau_{\mathrm{coh}}=6 \mathrm{fs}$ is obtained. Remarkably, the visibility, i.e. the mutual coherence, is not a monotonic function of the delay time between both partial beams. Instead, a minimum at about $7.4 \mathrm{fs}$ after the main maximum and a secondary maximum at about $12.3 \mathrm{fs}$ appear, symmetrically on both sides of the main maximum. In addition, a small but discernible increase of the visibility occurs at a delay around $40 \mathrm{fs}$. 

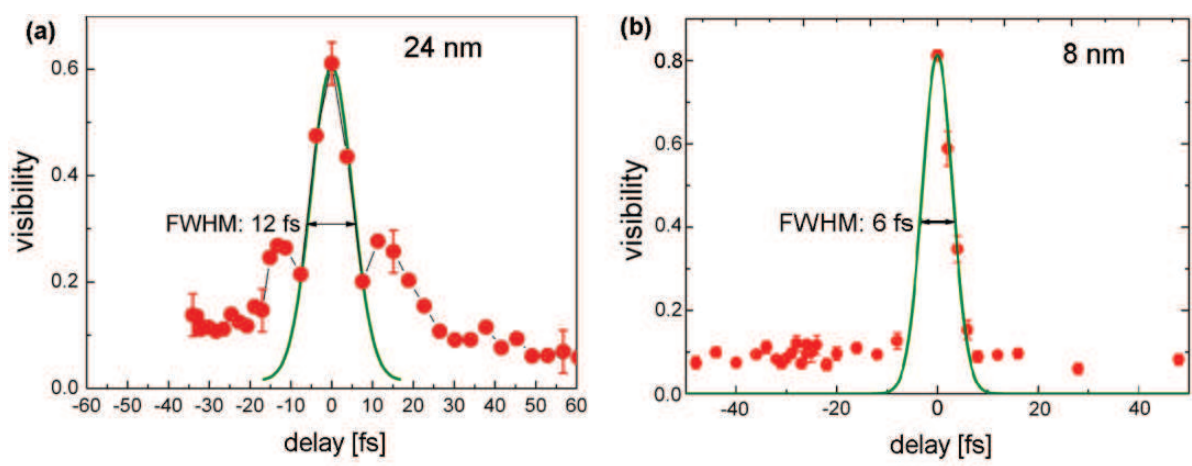

Fig. 12. Observed visibility (experimental data points: red dots) as a function of time delay for (a) $\lambda=24 \mathrm{~nm}$ and (b) $\lambda=8 \mathrm{~nm}$. The green line depicts a Gaussian function with a coherence time of (a) $\tau_{\mathrm{coh}}=6 \mathrm{fs}$ and (b) $\tau_{\mathrm{coh}}=3 \mathrm{fs}$, representing a single Fourier transform limited pulses.

Since the interferences were measured for independent single pulses of the FEL and then their visibilities averaged, this behavior of the temporal coherence function reflects an intrinsic feature of the FEL pulses at the time of the measurements. The radiation of SASE FELs consists of independently radiating transverse and longitudinal modes. In the time domain the radiation is emitted in short bursts with random phase relationship between the bursts. Time domain and spectral domain are related to each other via a Fourier transformation which leads to narrow spikes within the bandwidth of the undulator in the spectral domain, see also the calculated spectrum of a SASE FEL shown in Fig. 2. In the linear autocorrelation experiments shown in Fig. 12a (Mitzner et al., 2008) these independent modes can interact at longer time delays as a cross correlation. This behavior was found to be accountable for the nonmonotonous decay of the visibility. A second sub-pulse at $\Delta t=12 \mathrm{fs}$ and a weak third one at $\Delta \mathrm{t}$ $=40 \mathrm{fs}$ can be stated as a reason for this behavior. Figure 12 (b) shows the result from an analogous measurement at $\lambda=8 \mathrm{~nm}$. From a Gaussian fit with a FWHM of $6 \mathrm{fs}$ a coherence time of $\tau_{\mathrm{c}}=3 \mathrm{fs}$ is obtained. The non-monotonous decay that was discussed before for the 24 $\mathrm{nm}$ measurement is not apparent here.

Recently, a similar measurement also utilizing an autocorrelator that employs wave front beam splitters was performed for FLASH radiating at $\lambda=9.1 \mathrm{~nm}$ and $\lambda=33.2 \mathrm{~nm}$ (Schlotter et. al, 2010). These data were compared to Fourier transformed spectral bandwidth measurements obtained in the frequency domain by single-shot spectra. A good agreement with the measurements in the time domain was found. In addition to single shot exposures the temporal coherence was measured in the 15-pulse-per-train mode. Figure 13 shows the time delay dependence of the average visibility observed for two different wavelengths, $\lambda=$ $33.2 \mathrm{~nm}$ (single shot: black triangles; 15 bunches: red triangles) and $\lambda=9.6 \mathrm{~nm}$ (single shot: black squares; 15 bunches: red dots). In order to plot the data of both wavelengths into one graph the abscissa is given in $c \tau / \lambda$ which represents the number of periods of the lightwave. For the 15 bunch per train data a clearly lower coherence at longer timescales is observed than for the single shot data.

To explain this behavior we should take a look at a single point in the interference pattern. If a maximum of the intensity appears at this point for zero delay and for a path length differences $n \cdot \lambda$, minima will appear for $\frac{n}{2} \cdot \lambda$. The wavelength of the FEL radiation shows 


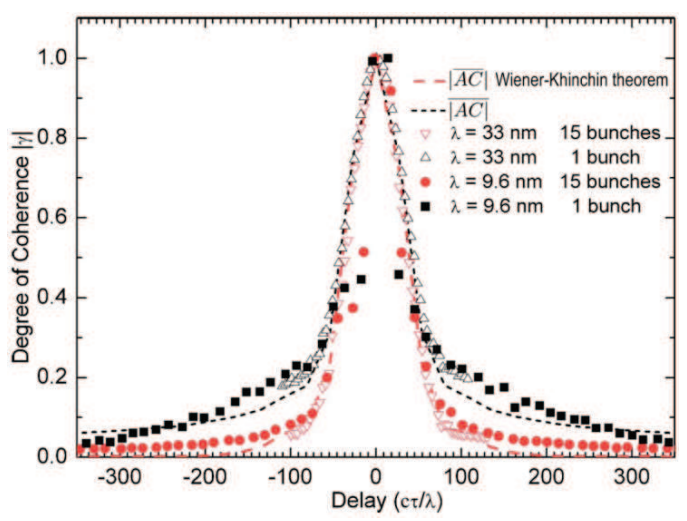

Fig. 13. The normalized degree of coherence $|\gamma|$ plotted versus the delay given in units of the wavelength. The dashed curve was calculated from spectral measurements at $33.2 \mathrm{~nm}$. Taken from reference (Schlotter et al., 2010).

small shot-to-shot fluctuations. Therefore for longer path length differences $(n \sim 50)$ a $\pi$ phase difference occurs for different wavelengths $\lambda_{k}$. At the same point of the detector the interference pattern corresponding to a wavelength $\lambda_{1}$ may now show a maximum while the interference pattern corresponding to a wavelength $\lambda_{2}$ shows a minimum. Thus, the visibility appears blurred, when $\mathrm{k}=15$ bunches with slightly different wavelengths form interference patterns before the read-out of the detector.

\subsection{Coherence enhancement through seeding}

An essential drawback of SASE FEL starting from shot noise is the limited temporal coherence. Therefore, the improvement of the temporal coherence is of great practical importance. One idea to overcome this problem was presented by Feldhaus et al. (Feldhaus et al., 1997). The FEL described consists of two undulators and an X-ray monochromator located between them (see Fig. 14). The first undulator operates in the linear regime of amplification and starts from noise. The radiation output has the usual SASE properties with significant shot-to-shot fluctuations. After the first undulator the electron beam is guided through a by-pass, where it is demodulated. The light pulse on the other hand is monochromatized by a grating. At the entrance of the second undulator the monochromatic X-ray beam is recombined with the demodulated electron beam, thereby acting as a seed for the second undulator. For this purpose, the electron micro-bunching induced in the first undulator must be destroyed, because this electron micro-bunching from the first undulator corresponds to shot noise that was amplified. The degree of micro-bunching can thus be characterized by the power of shot noise which has the same order of magnitude as the output power of the FEL. When the radiation now passes the monochromator only a narrow bandwidth and thus only a small amount of the energy is transmitted. Thus at the entrance of the second undulator a radiation-signal to shot-noise ratio much larger than unity has to be provided. This can be achieved because of the finite value of the natural energy spread in the beam and by applying a special design of the electron by-pass.

At the entrance of the second undulator the radiation power from the monochromator then dominates over the shot noise and the residual electron bunching, such that the second stage of the FEL amplifier will operate in the steady-state regime when the input signal 


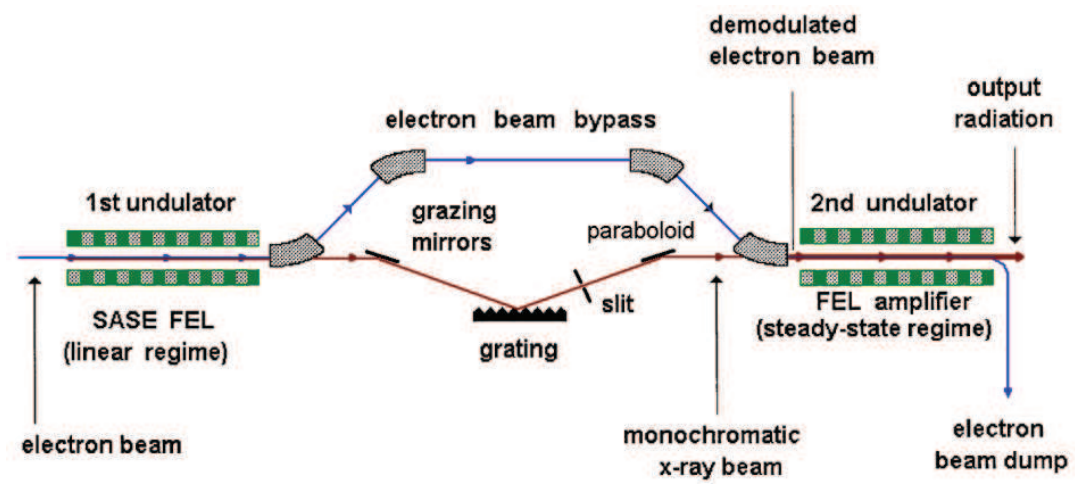

Fig. 14. Principal scheme of a single-pass two-stage SASE X-ray FEL with internal monochromator; after (Saldin et al., 2000a).

bandwidth is small with respect to the FEL amplifier bandwidth. The second undulator will thus amplify the seed radiation. The additional benefits derived from this configuration are superior stability, control of the central wavelength, narrower bandwidth, and much smaller energy fluctuations than SASE. Further, it is tunable over a wide photon energy range, determined only by the FEL and the grating.

An alternative approach is based on seeding with a laser, see ref. (Yu et al., 1991, 2000). Such a scheme has been applied at the Deep Ultraviolet FEL (DUV FEL) at the National Synchrotron Light Source (NSLS) of Brookhaven National Laboratory (BNL) (Yu et al., 2003). The set-up is shown in Fig. 15. In high-gain harmonic generation (HGHG) a small energy modulation is imposed on the electron beam by its interaction with a seed laser (1) in a short undulator (8) (the modulator) tuned to the seed wavelength $\lambda$. The laser seed introduces an energy modulation to the electron bunch. In a dispersive three-dipole magnetic chicane (9) this energy modulation is then converted into a coherent longitudinal density modulation. In a second long undulator (10) (the radiator), which is tuned to the $n$th odd harmonic of the seed frequency, the microbunched electron beam emits coherent radiation at the harmonic frequency $n \lambda$, which is then amplified in the radiator until saturation is reached. The modulator (resonant at $\lambda=800 \mathrm{~nm}$ ) of the DUV FEL is seeded by an $800 \mathrm{~nm}$ CPA Ti:sapphire laser (pulse duration: $9 \mathrm{ps}$ ). This laser drives also the rf gun of the photocathode producing an electron bunch of 1 ps duration. In this way an inherent synchronization between the electron bunches and the seeding pulses is achieved. The output properties of the HGHG FEL directly maps those of the seed laser which can show a high degree of temporal coherence. In the present case the output HGHG radiation shows a bandwidth of $0.23 \mathrm{~nm}$ FWHM (corresponding to $\sim 0.1 \%$ ), an energy fluctuation of only $7 \%$ and a pulse length of 1 ps (equal to the electron bunch length) when the undulator is seeded with an input seed power of $P_{\text {in }}=30$ MW. The bandwidth within a $1 \mathrm{ps}$ slice of the chirped seed is $0.8 \mathrm{~nm}$ (corresponding to $0.1 \%$ bandwidth) and the chirp in the HGHG output is expected to be the same, i.e., $0.1 \% \cdot 266 \mathrm{~nm}=$ $0.26 \mathrm{~nm}$. This is consistent with a bandwidth of $\Delta \lambda=0.23 \mathrm{~nm}$ [FWHM] experimentally observed. A Fourier-transform limited flat-top 1 ps pulse would have a bandwidth of $\Delta \lambda=0.23$ $\mathrm{nm}$ and a 1 ps (FWHM) Gaussian pulse would have a bandwidth of $\Delta \lambda=0.1 \mathrm{~nm}$. Besides the high degree of temporal coherence a further advantage compared to a SASE FEL is the reduced shot-to-shot fluctuations of the output radiation if the second undulator operates in 
saturation. A similar scheme will also be applied at the FERMI FEL at Elettra that will start operation in 2011 providing wavelengths down to $\lambda=3 \mathrm{~nm}$ for the fundamental and $\lambda=1 \mathrm{~nm}$ for the third harmonic (Allaria et al., 2010).

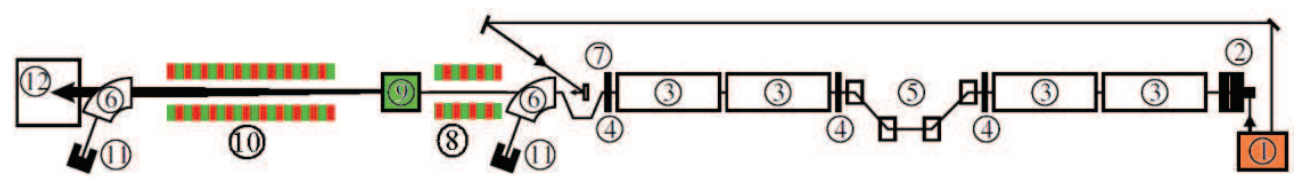

Fig. 15. The NSLS DUV FEL layout. 1: gun and seed laser system; 2: rf gun; 3: linac tanks; 4: focusing triplets; 5: magnetic chicane; 6: spectrometer dipoles; 7: seed laser mirror; 8 : modulator; 9: dispersive section; 10: radiator; 11: beam dumps; 12: FEL radiation measurements area. After reference (Yu et al., 2003).

Another possibility to generate coherent radiation from an FEL amplifier is seeding with high harmonics $(\mathrm{HH})$ generated by an ultrafast laser source whose beam properties are simple to manipulate, see reference (Sheehy et al., 2006; Lambert et al., 2008). In this way extremely short XUV pulses are obtained, down to a few femtoseconds. Such a scheme was applied at the Spring-8 compact SASE source (Lambert et al., 2008) and is depicted schematically in Fig. 16.

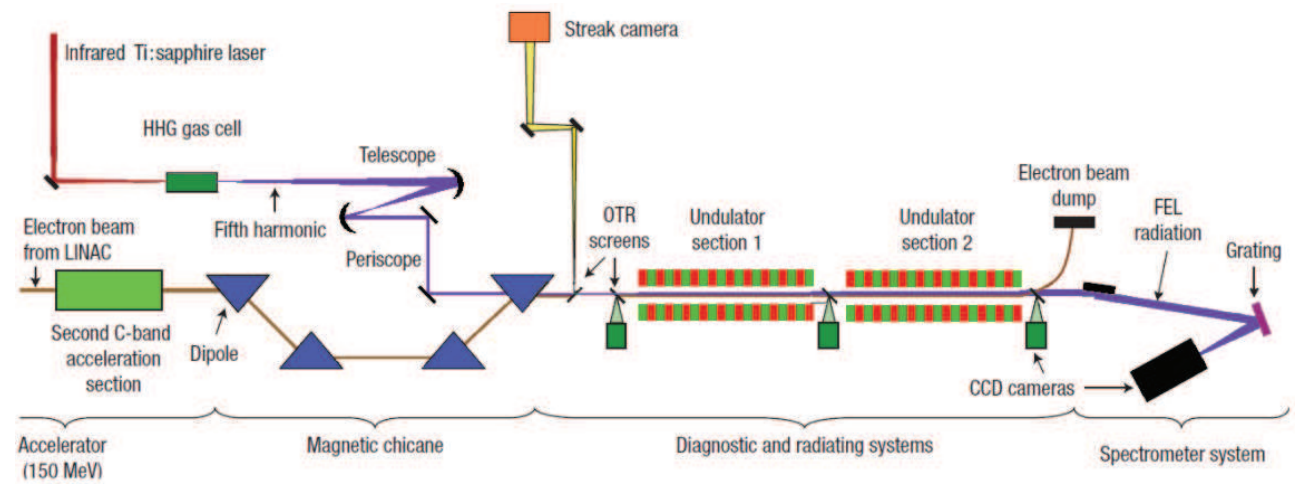

Fig. 16. Experimental setup for HHG seeding of the Spring-8 Compact SASE source, after (Lambert et al., 2008).

A Ti:sapphire laser $(800 \mathrm{~nm}, 20 \mathrm{~mJ}, 100 \mathrm{fs}$ FWHM, $10 \mathrm{~Hz}$ ) that is locked to the highly stable $476 \mathrm{MHz}$ clock of the accelerator passes a delay line that is necessary to synchronize the HHG seed with the electron bunches. For this purpose a streak camera observes the $800 \mathrm{~nm}$ laser light and the electron bunch signal from an optical transition radiation (OTR) screen. The beam is then focused into a xenon gas cell in order to produce high harmonics. Using a telescope and periscope optics the HHG seed beam is spectrally selected, refocused and spatially and temporally overlapped with the electron bunch (150 $\mathrm{MeV}, 1$ ps FWHM, $10 \mathrm{~Hz}$ ) in the two consecutive undulator sections 1 and 2 . Both undulators are tuned to $\lambda=160 \mathrm{~nm}$, corresponding to the fifth harmonic of the laser. The beam position is monitored on optical transition radiation (OTR) screens. The output radiation is characterized with an imaging spectrometer for different seeding pulse energies between $0.53 \mathrm{~nJ}$ and $4.3 \mathrm{~nJ}$ per pulse. 
Figure 17 shows the spectra of the unseeded undulator emission (purple, enlarged 35 times), the HHG seed (yellow, enlarged 72 times) and the seeded radiation output (green) for a seed pulse energy of $4.3 \mathrm{~nJ}$. A spectral narrowing for the seeded output radiation and a significant shift to longer wavelengths compared with the seed radiation is obvious. The measured relative spectral widths of the seeded FEL are reduced compared to the unseeded one from $0.54 \%$ to $0.46 \%$ ( $0.53 \mathrm{~nJ}$ seed) and from $0.88 \%$ to $0.44 \%$ ( $4.3 \mathrm{~nJ}$ seed). A similar narrowing is observed for the spectra of the third $(\lambda=53.55 \mathrm{~nm})$ and fifth harmonic $(\lambda=32.1 \mathrm{~nm})$.

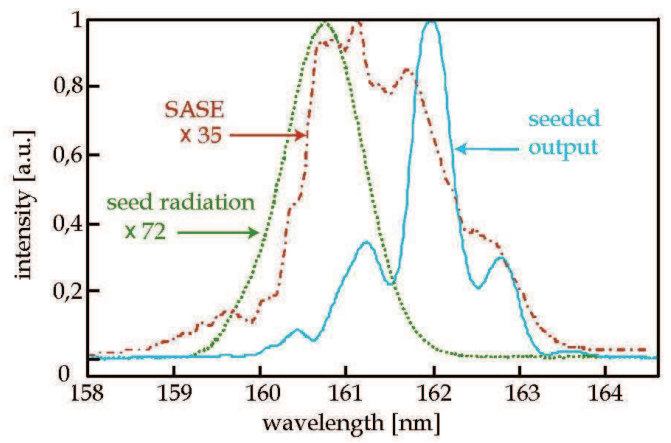

Fig. 17. Experimentally obtained spectra of the FEL fundamental emission $(\lambda=160 \mathrm{~nm})$ : SASE (red), seed radiation (green) and seeded output (blue), after (Lambert et al., 2008).

For a fully coherent seed pulse the seeded FEL should also show a high temporal coherence which, however, is not yet experimentally confirmed. The pulse should then also show a duration close to the Fourier transform limit. From the measured spectral width of $\Delta \lambda=0.74$ $\mathrm{nm}$ (for $0.53 \mathrm{~nJ}$ seed) one might conclude a Fourier transform limited duration of $57 \mathrm{fs}$. Currently several facilities using HHG as a seed source are proposed or under construction e.g. references (McNeil et al., 2007; Miltchev et al., 2009).

\section{Temporal coherence of high-order harmonic generation sources}

The generation of high-order harmonics of a short laser pulse in a gas jet has attracted a lot of attention since the first discovery in the late 1980s (McPherson et al., 1987; Ferray et al., 1988; Li et al., 1989). High harmonic radiation has become a useful short-pulse coherent light source in the XUV spectral regime (Haarlammert \& Zacharias, 2009; Nisoli \& Sansone, 2009). By focussing an intense femtosecond laser pulse into rare gases odd order high harmonics of the original laser frequency can be generated.

This can be explained in terms of the three step model (Corkum, 1993; Kulander et al., 1993; Lewenstein et al., 1994). The focused pumping laser beam typically has intensities of more than $10^{13} \mathrm{~W} / \mathrm{cm}^{2}$, which is in the order of the atomic potential. This leads to a disturbance of the atomic potential of the target atoms allowing the electron to tunnel through the remaining barrier, see Fig. 18a. Figure 18b shows how the electron is then accelerated away from the atom core by the electric field of the driving laser lightwave. After half an optical cycle the direction of the driving laser field reverses and the electron is forced to turn back to the core. There, a small fraction of the electrons recombine with the ion, and the energy that was gained in the accelerating processes before plus the ionization energy $\mathrm{I}_{\mathrm{P}}$ is emitted as light, see Fig. 18c. When the electrons turn back to the core they can basically follow two 

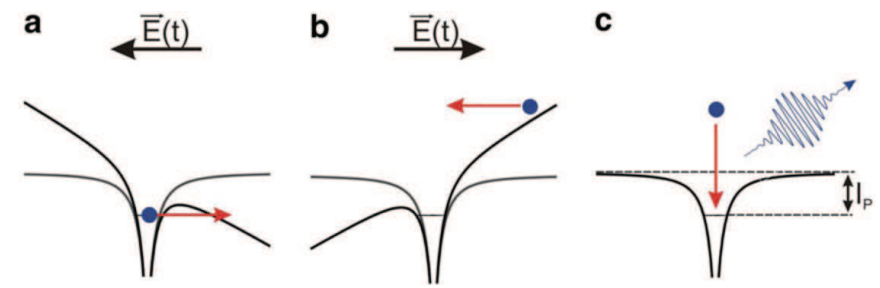

Fig. 18. Illustration of the three step model for high harmonic generation. (a) deformation of the atomic potential and tunnel ionization of the target atoms (b) acceleration of the free electrons in the laser electric field (c) recombination and photon emission.

different trajectories, a short one and a long one, respectively. The short trajectory shows an excursion time close to half an optical cycle, whereas the long trajectory takes slightly less than the whole optical period. Both of them show different phase properties with respect to the dipole moment of the particular harmonic. The phase of the short trajectory does not significantly vary with the laser intensity as opposed to the phase of the long trajectory that varies rapidly with the laser intensity (Lewenstein et al., 1995; Mairesse et al., 2003). The energy acquired by the electron in the light field corresponds to the ponderomotive energy $U_{p}$

$$
U_{p}=e^{2} E_{o}^{2} / 4 m_{e} \omega^{2}
$$

Here $\mathrm{E}_{0}$ denotes the electric field strength, e the elementary charge, $\mathrm{m}_{\mathrm{e}}$ the electron mass and $\omega$ the angular frequency. The maximum photon energy emitted, the cut-off energy, is given by

$$
E_{\text {cutoff }}=3.17 \cdot U_{\text {pon }}+I_{p},
$$

where $\mathrm{I}_{\mathrm{p}}$ denotes the ionization potential of the atom.

A theoretical study of the coherence properties of high order harmonics generated by an intense short-pulse low-frequency laser is presented particularly for the $45^{\text {th }}$ harmonic of a $825 \mathrm{~nm}$ wavelength laser (Salières, L'Huillier \& Lewenstein, 1995). First, the generation of the radiation by a single atom is calculated by means of a semi-classical model (Lewenstein et al., 1994). Phase and amplitude of each harmonic frequency are evaluated and then in a second step propagated in terms of the slowly varying amplitude approximation (L'Huillier et al., 1992). Harmonic generation is optimized when the phase-difference between the electromagnetic field of the driving laser and the electromagnetic field of the output radiation is minimized over the length of the medium. At this point phase-matching is achieved. It is shown that the coherence properties and consequently the output of the harmonics can be controlled and optimized by adjusting the position of the laser focus relative to the nonlinear medium.

Bellini et al. investigated experimentally the temporal coherence of high-order harmonics up to the 15th order produced by focusing $100 \mathrm{fs}$ laser pulses into an argon gas jet (Bellini et al., 1998; Lyngå et al., 1999). The visibility of the interference fringes produced when two spatially separated harmonic sources interfere in the far-field was measured as a function of time delay between the two sources. The possibility to create two phase-locked HHG sources that are able to form an interference pattern in the far-field had been demonstrated earlier (Zerne et. al., 1997). A high transverse coherence that is necessary for two beams to interfere under an angle had been proven by a Youngs double-slit set-up (Ditmire et al., 1996). The experimental set-up used for the coherence measurements is shown in Fig. 19. 


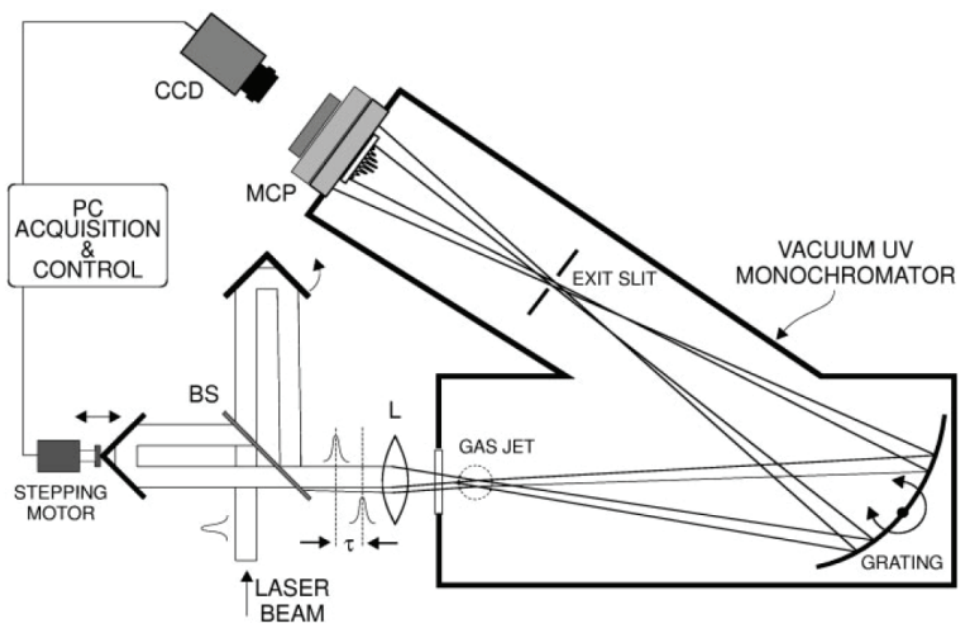

Fig. 19. Experimental setup for the measurement of the temporal coherence of high-order harmonics. BS is a broadband $50 \%$ beam splitter for $800 \mathrm{~nm}$. L is the lens used to focus the two pulses, separated by the time delay $\tau$, into the gas jet. Taken from reference (Bellini et al., 1998).

The laser used was an amplified Ti:sapphire system delivering $100 \mathrm{fs}$ pulses with $14 \mathrm{~nm}$ spectral width centered around $790 \mathrm{~nm}$ at a $1 \mathrm{kHz}$ repetition rate and with an energy up to $0.7 \mathrm{~mJ}$. A Michelson interferometer placed in the path of the laser beam produced pairs of near infrared pump pulses which had equal intensities and whose relative delay could be accurately adjusted by means of a computer controlled stepping motor. The beams were then apertured down and focused into a pulsed argon gas jet. In order to avoid interference effects in the focal zone and to prevent perturbations of the medium induced by the first pulse one arm of the interferometer was slightly misaligned. Thus the paths of the two pulses were not perfectly parallel to each other and formed a focus in two separate positions of the jet. Both pulses then interacted with different Ar ensembles and produced harmonics as two separate and independent sources that may interfere in the far field. Behind the exit slit of a monochromator spatially overlapped beams were detected on the sensitive surface of a MCP detector coupled to a phosphor screen and a CCD camera.

To determine the temporal coherence of the high-order harmonics the time delay between both generating pulses was varied in steps of 5 or $10 \mathrm{fs}$ and successive recordings of the interference patterns were taken. The fringe visibility $\mathrm{V}$ is calculated according to equation 4 for the different delays $\Delta t$ and for different points in the interference pattern in order to analyze the temporal coherence properties spatially for inner and outer regions of the beam. The coherence time was obtained as the half width at half maximum of the curve shown in Fig. 20.

The coherence times measured at the center of the spatial profile varied from 20 to $40 \mathrm{fs}$, relatively independently of the harmonic order. In the outer region a much shorter coherence time is observed. This can be explained when the different behavior of the phases of the long and the short trajectory due to the laser intensity is taken into account. In a simulation the contributions of these trajectories are examined. Because the long trajectory shows a rapid variation of the dipole phase that leads to a strong curvature of the phase 


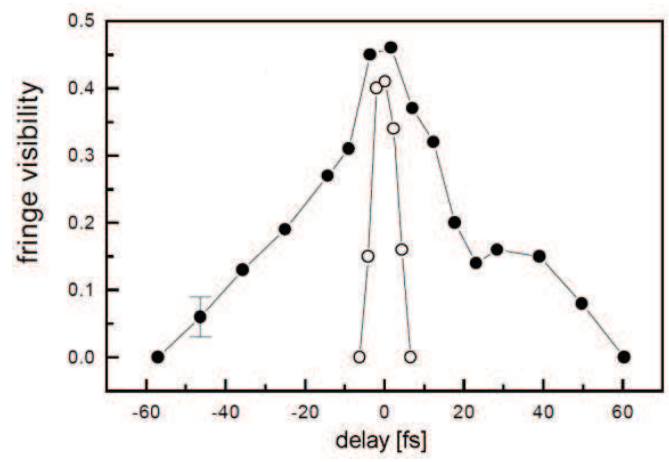

Fig. 20. Visibility curves as a function of the delay for the 15th harmonic, for the inner (full symbols) and outer (open symbols) regions. Taken from (Bellini et. al., 1998).

front, the radiation emitted from this process has a short coherence time due to the chirp caused by the rapid variation of the phase during the pulse. As opposed, the short trajectory shows a long coherence time. Since the radiation emerging from the short trajectory is much more collimated than that from the long trajectory its contribution to the outer part of the observed interference pattern is much lower than that of the latter. At this point it is necessary to emphasize that in this experiment the temporal coherence of two phase-locked HHG sources is evaluated, where the time delay is introduced between the partial beams of the driving Ti:sapphire laser. Therefore the two XUV pulses have to be assumed to be identical.

Hemmers and Pretzler presented an interferometric set-up operating in the XUV spectral range (Hemmers \& Pretzler, 2009). The interferometer consisted of a combination of a double pinhole (similar to Young's double slit) and a transmission grating. In the case of a light source consisting of discrete spectral lines, it allows to record interferograms for multiple colors simultaneously. The experimental setup is shown in Fig. 21.

The pinholes were mounted such that a defined rotation around the beam axis was possible. A transmission grating placed behind the pinholes dispersed the radiation spectrally. Spectra were recorded by a CCD camera placed at a distance of $L=135 \mathrm{~cm}$ from the pinholes. This set-up is suitable to be used as a spectrometer with the double pinhole as a slit. The spectral resolution is determined by the pinhole diffraction, which creates Airy spots in the far-field. With the described geometry this leads to a spectral resolution in the range of $\Delta \lambda=0.3 \mathrm{~nm}$ at a wavelength of $\lambda=20 \mathrm{~nm}$, sufficient to separate individual odd harmonics with spectral separation of about $1 \mathrm{~nm}$ in that spectral range. Furthermore, the combination of a rotatable double pinhole and a transmission grating acts as a spectrally resolved Young's double slit interferometer with variable slit spacing. The time delay between the partial beams was realized in the following manner: a varying path difference between the two interfering beams was achieved by rotating the double pinhole around the grating normal. As illustrated in Fig. 21, the path difference in the beams diffracted into first order by the grating varies as

$$
\Delta s=D^{*} \sin \beta^{*} \sin \gamma=D^{*} \sin \beta^{*} N \lambda .
$$

Here $\gamma$ denotes the diffraction angle and $\beta$ the rotation angle of the pinholes with respect to the grating. 


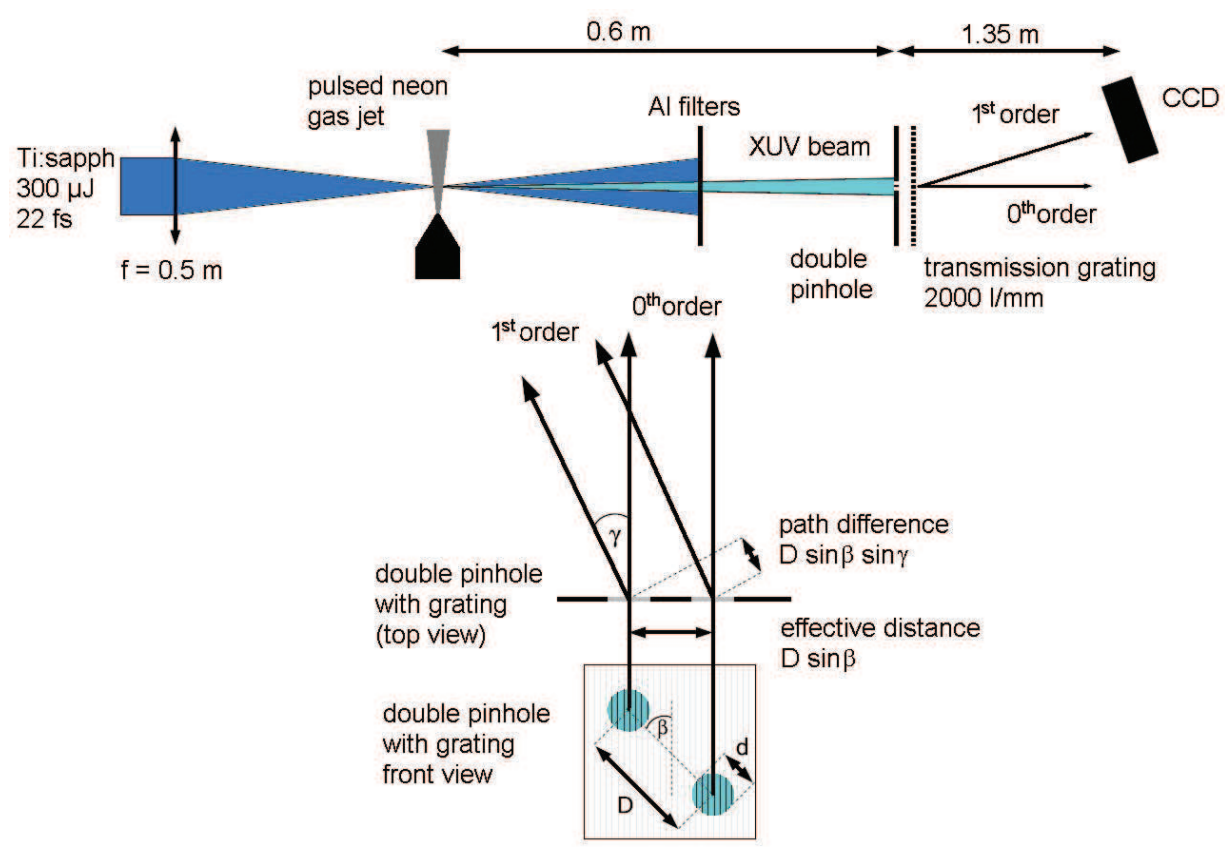

Fig. 21. Experimental set-up of high harmonics generation and the rotatable pinhole interferometer; after (Hemmers \& Pretzler, 2009)

This allowed the variation of the path length difference $|\Delta s|$ between zero $(\beta=0$ : pinholes perpendicular to the dispersion direction) and $200 \cdot \lambda=16,7 \mathrm{fs}$ at $\lambda=25 \mathrm{~nm}(\beta= \pm \pi / 2)$ with respect to the given geometrical parameters. When the two diffracted Airy disks overlap partially an interference pattern occurs on the detector for each single harmonic if the light is sufficiently coherent. The visibility $\mathrm{V}$ for different delays was then calculated according to equation 4 .

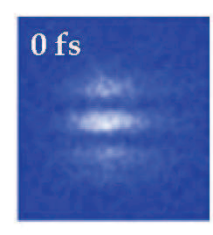

(a)
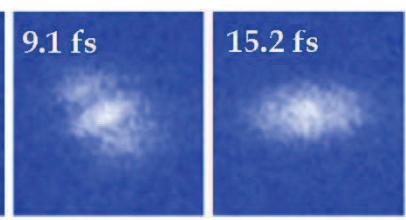

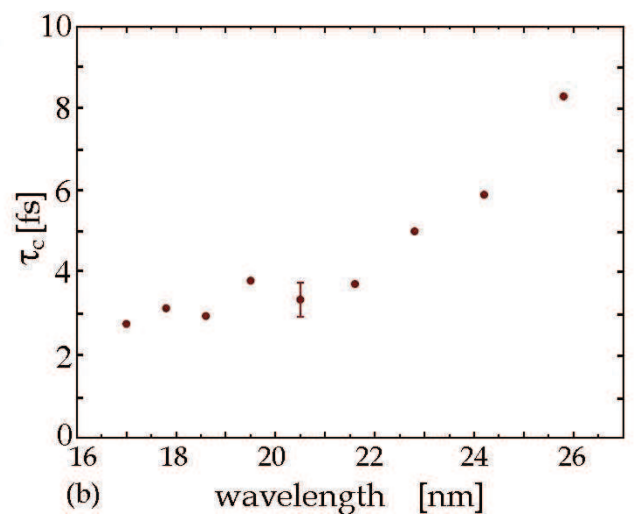

Fig. 22. (a) Interference patterns for different time delays. (b) Coherence times $\tau_{\mathrm{c}}$ for the harmonics H17 - H 26; after reference (Hemmers \& Pretzler, 2009). 
The temporal coherence lengths of the single harmonics (H47 - H31, $\lambda=17-25 \mathrm{~nm}$ ) generated by a Ti:sapphire laser focused into a neon gas jet were determined. The results are shown in Fig. 22. Interference patterns for different path length differences show that the high visibility that occurs for zero delay degrades for $\Delta \mathrm{s}=2.74 \mu \mathrm{m}$ and then vanishes at $\Delta \mathrm{s}=$ $4.57 \mu \mathrm{m}$ (Fig. 22a). An observed decrease of the coherence length from $2.5 \mu \mathrm{m}$ at $\lambda=25 \mathrm{~nm}$ to $0.8 \mu \mathrm{m}$ at $\lambda=17 \mathrm{~nm}$ corresponds to coherence times of $\mathrm{t}_{\mathrm{c}}=8.3 \mathrm{fs}$ and $\mathrm{t}_{\mathrm{c}}=2.7 \mathrm{fs}$, respectively.

\section{Summary}

In this article recent developments in research on coherence properties of free electron lasers and high-harmonic sources in the xuv and soft $x$-ray spectral regime were reviewed. Theoretical results applying 1D- and 3D-numerical simulations for SASE FEL yield good spatial coherence but rather poor temporal coherence which is confirmed by experimental studies. Promising seeding methods for the improvement of the coherence properties and the stability of the output radiation power of free electron lasers were discussed. Several FEL user facilities are nowadays proposed or under construction applying such sophisticated techniques. The first FEL utilizing a HHG scheme, SCSS at Spring-8 in Japan, has succesfully proven this principle in the $x$-ray spectral regime. A scheme making use of a HHG light source for the seeding of an undulator is now applied at S-FLASH. The outstanding coherence properties of such light sources discussed in this article predestine HHG for the seeding of FEL. Nevertheless FEL based on SASE are established light sources in the XUV and soft $\mathrm{x}$-ray spectral regime. This technique will also be employed for upcoming projects such as the European XFEL. For the latter also a pulse splitting and delay unit (autocorrelator) similar to that one at FLASH presented in this article is now under construction. With this device a measurement of the coherence properties as well as jitter free hard x-ray pump - x-ray probe experiments will be possible.

\section{References}

Ackermann, W. et al. (2007). Operation of a free-electron laser from the extreme ultraviolet to the water window. Nature Photonics, 1, (2007) 336-342

Allaria, E.; Callegari, C.; Cocco, D.; Fawly, W.M.; Kiskinova, M.; Masciovecchio, C. \& Parmigiani, F. (2010). The FERMI@Elettra free-electron-laser source for coherent $x-$ ray physics: photon properties, beam transport system and applications. New Journal of Physics, 12, (2010) 075022

Altarelli, M.; Brinkmann, R.; Chergui, M.; Decking, W.; Dobson, B.; Düsterer, S.; Grübel, G.; Greaff, G.; Graafsma, H.; Haidu, J.; Marangos, J.; Pflüger, J.; Redlin, H.; Riley, D.; Robinson, I.; Rossbach, J.; Schwarz, A.; Tiedtke, K.; Tschentscher, T.; Vartaniants, I.; Wabnitz, H.; Weise, H.; Wichmann, R.; Witte, K.; Wolf, A.; Wulff, M. \& Yurkov, M. (2006). The European X-Ray Free-Electron Laser Technical Design Report. DESY XFEL Project Group, European XFEL Project Team, Deutsches Elektronen-Synchrotron, ISBN 978-3-935702-17-1, Hamburg

Bellini, M.; Lyngå, M.; Tozzi, A.; Gaarde, M.B.; Hänsch, T.W.; L'Huillier, A. \& C.-G. Wahlström (1998). Temporal Coherence of Ultrashort High-Order Harmonic Pulses. Physical Review Letters, 81, (1998) 297-300

Bonifacio, R.; Pellegrini, C. \& Narducci, L.M. (1984). Collective instabilities and high-gain regime in a free electron laser. Optics Communications, 50, (1984) 373-378 
Bonifacio, R.; De Salvo, L.; Pierini, P.; Pivvella, N.; Pellegrini , C. (1994a). A study of linewidth, noise and fluctuations in a FEL operating in SASE. Nuclear Instruments and methods in Physics Research A, 341, (1994) 181-185

Bonifacio, R.; De Salvo, L.; Pierini, P.; Pivvella, N.; Pellegrini , C. (1994b). Spektrum, Temporal Structure, and Fluctuations in a High-Gain Free-Electron Laser starting from Noise. Physical Review Letters, 73, (1994) 70-73

Chapman, H.N.; Barty, A.; Bogan,M. J.; Boutet, S.; Frank, M.; Hau-Riege, S. P.; Marchesini, S.; Woods, B.W.; Bajt, S.; Benner, W.H.; London, R.A.; Plönjes, E.; Kuhlmann, M.; Treusch, R.; Düsterer, S.; Tschentscher, T.; Schneider, J. R.; Spiller, E.; Möller, T.; Bostedt, C.; Hoener, M.; Shapiro, D.A.; Hodgson, K. O.; van der Spoel, D.; Burmeister, F.; Bergh, M.; Caleman, C.; Huldt, G.; Seibert, M.M.; Maia, F. R. N. C.; Lee, R. W.; Szöke, A.; Timneanu, N. \& Hajdu J. (2006). Femtosecond diffractive imaging with a soft-X-ray free-electron laser. Nature Physics, 2, (2006) 839 - 843

Corkum P. (1993). Plasma perspective on strong-field multiphoton ionisation. Physical Review Letters, 71, (1993) 1994-1997

Deacon, D.A.G.; Elias, L.R.; Madey, J.M.J.; Ramian, G.J.; Schwettman, H.A. \& Smith, T.I. (1977). First Operation of a Free-Electron Laser. Physical Review Letters, 38, (1977), 892-894

Deutsches Elektronen-Synchrotron Homepage (2010). Record wavelength at FLASH - First lasing below 4.5 nanometres at DESY's free-electron laser, Hamburg (June 2010), http://zms.desy.de/news/desy_news/2010/flash_record/index_eng.html

Ditmire, T.; Gumbrell, E. T.; Smith, R. A.; Tisch, J. W. G.; Meyerhofer, D. D. \& Hutchinson M.H.R. (1996). Spatial Coherence Measurement of Soft X-Ray Radiation Produced by High Order Harmonic Generation. Physical Review Letters, 77,(1996) 4756-4758

Eisebitt S.; Lüning, J.; Schlotter, W. F.; Lörgen, M.; Hellwig, O.W.; Eberhardt W. \& Stöhr J. (2004). Lensless imaging of magnetic nanostructures by X-ray spectro-holography. Nature, 432, (2004) 885-888

Emma, P.; Akre, R.; Arthur, J.; Bionta, R.; Bostedt, C; Bozek, J.; Brachmann, A.; Bucksbaum, P.;Coffee, R.; Decker, F.-J.; Ding, Y.; Dowell, D.; Edstrom, S.; Fisher, A.; Frisch, J.; Gilevich, S.; Hastings, J.; Hays, G.; Hering, Ph.; Huang, Z. ; Iverson, R.; Loos, H.; Messerschmidt, M.; Miahnahri1, A.; Moeller, S.; Nuhn, H.-D. ; Pile, G.; Ratner, D.; Rzepiela, J.; Schultz, D.; Smith, T.; Stefan, P.; Tompkins, H.; Turner, J.; Welch, J.; White, W.; Wu, J.; Yocky, G. \& Galayda, J. (2010). First lasing and operation of an ångstrom-wavelength free-electron laser. Nature photonics, 4, (2010) 641-647

Feldhaus, J.; Saldin, E.L.; Schneider, J.R.; Schneidmiller, E.A.; M.V. Yurkov (1997). Possible application of X-ray optical elements for reducing the spectral bandwidth of an Xray SASE FEL. Optics Communications, 140, (1997) 341-352

Feldhaus, J ; Arthur, J \& Hastings J. B. (2005). X-ray free electron lasers. Journal of Physics B: Atomic, Molecular and Optical Physics, 38, (2005) 799-899

Ferray, M; L'Huillier, A; Li, X; Lompre, L; Mainfray, G \& Manus, C. (1988). Multipleharmonic conversion of 1064-nm radiation in rare-gases, Journal of Physics B: Atomic, Molecular and Optical Physics, 21, (1988) L31-L35

Geloni, G.; Saldin, E.; Schneidmiller E.; Yurkov, M. (2008). Transverse coherence properties of X-ray beams in third-generation synchrotron radiation sources. Nuclear instruments and Methods in Physics Research A: Accelerators, Spectrometers, Detectors and Associated Equipment, 588, (2008) 463-493 
Goodman, J.W. (2000). Statistical Optics. Wiley \& Sons, ISBN 047139916 7, New York

Haarlammert, T. \& Zacharias, H. (2009). Application of high-harmonic radiation in surface science. Current Opinion in Solid State and Materials Science, 13, (2009) 13-27

Hemmers, D. \& Pretzler, G. (2009). Multi-color XUV Interferometry Using High-Order Harmonics. Applied Physics B: Lasers and Optics, 95, (2009) 667-674

Ischebeck, R.; Feldhaus, J.; Gerth, Ch.; Saldin, E.; Schmüser, P.; Schneidmiller, E.; Steeg, B.; Tiedtke, K. ; Tonutti, M.; Treusch, R. \& Yurkov M. (2003). Study of the transverse coherence at the TTF free electron laser, Nuclear Instruments and Methods in Physics Research A, 507, (2003) 175-180

Kondratenko, A.M. \& Saldin, E.L. (1980). Generation of coherent radiation by a relativistic electron beam in an ondulator. Particle Accelerators, 10, (1980) 207-216

Kuhlmann, M.; Caliebe, W.; Drube, W. \& Schneider, J. R. (2006). HASYLAB Annual Report, Part I, (2006) 213

Kulander, K; Schafer, K \& Krause, J. (1993). Super-intense laser-atom physics. Plenum Press, New York and London

Lambert, G.; Hara, T.; Garzella, D.; Tanikawa, T.; Labat, M.; Carre, B.; Kitamura, H.; Shintake, T.; Bougeard, M.; Inoue, S.; Tanaka, Y.; Salieres P., Merdju, H.; Chubar, O., Gobert, O.; Tahara, K. \& Couprie M.-E. (2008). Injection of harmonics generated in gas in a free-electron laser providing intense and coherent extreme-ultraviolet light. Nature Physics, 4, (2008) 296-300

Lewenstein, M.; Balcou, P.; Ivanov, M.; L'Huillier, A. \& Corkum P. (1994). Theory of highharmonic generation by low frequency laser fields. Physical Review A, 49, (1994), 2117-2132

L'Huillier, A.; Balcou, P.; Candel, S.; Schafer, K.J. \& Kulander, K.C. (1992). Calculations of high-order harmonic-generation processes in xenon at $1064 \mathrm{~nm}$. Physical Review A, 46, (1992) 2778 - 2793

Li, X; L'Huillier, A.; Ferray, M.; Lompre, L.; Manfray, G. \& Manus, C. (1989); Multipleharmonic generation in rare gases at high laser intensity, Physical Review A, 39 (1989) 5751-5761

Lyngå, C.; Gaarde, M.B.; Delfin, C.; Bellini, M.; Hänsch, T.W.; L'Huillier, A. \& Wahlström, C.-G. (1999). Temporal coherence of high-order harmonics, Physical Review A, 60, (1999) 4823-4830

Mairesse, Y.; de Bohan, A.; Frasinski, L. J.; Merdji, H.; Dinu, L. C.; Monchicourt, P.; Breger, P.; Kovačev, M.; Taïeb, R.; Carré, B.; Muller, H. G.; Agostini, P. \& Salières, P. (2003). Attosecond Synchronization of High-Harmonic Soft X-rays, Science, 302, (2003) 1540-1543

Madey, J.M.J. (1971). Stimulated Emission of Bremsstrahlung in a Periodic Magnetic Field. Journal of Applied Physics, 42, (1971) 1906-1930

Mandel, L. \& Wolf, E. (1995). Optical coherence and quantum optics, Cambridge university press, ISBN 052141711 2, Cambridge

McNeil, B.W.J.; Clarke, J.A., Dunning D.J.; Hirst, G.J.; Owen, H.L.; Thompson N.R.; Sheehy, B. \& Williams, P.H. (2007). An XUV-FEL amplifier seeded using high harmonic generation. New Journal of Physics, 9, (2007) 1367-2630

McNeil, B. (2009); First light from hard X-ray laser, Nature Photonics, 3, (2009) 375-377 
McPherson, A; Gibson, G; Jara, H; Johann, U; Luk, T; McIntyre, I; Boyer, K. \& Rhodes, C.K. (1987). Studies of multiphoton production of vacuum ultraviolet-radiation in raregases. Journal of the Optical Society of America B, 4, (1987) 595-601

Miltchev, V.; Azima, A.; Bödewadt, J.; Curbis, F.; Drescher, M.; Delsim-Hashemi, H.; Maltezopoulos, T.; Mittenzwey, A.; Rossbach, J.; Tarkeshian, R.; Wieland, M., Düsterer, S.; Feldhaus, J.; Laarmann, T.; Schlarb, H.; Meseck, A.; Khan, S. \& Ischebeck, R. (2009). Technical design of the XUV seeding experiment at FLASH. Proceedings of FEL, pp. 503-506, Liverpool, UK

Mitzner, R.; Siemer, B.; Neeb, M.; Noll, T.; Siewert, F.; Roling, S.; Rutkowski, M.; Sorokin, A. A.; Richter, M.; Juranic, P.; Tiedtke, K.; Feldhaus, J.; Eberhardt, W. \& Zacharias H.(2008), Spatio-temporal coherence of free electron laser pulses in the soft x-ray regime, Optics Express, 16, (2008) 19909-19919

Mitzner, R.; Sorokin, A.A.; Siemer, B.; Roling, S.; Rotkowski, M.; Zacharias, H.; Neeb, M.; Noll, T.; Siewert, F.; Eberhardt, W.; Richter, M.; Juranic, P.; Tiedtke, K. \& Feldhaus, J. (2009). Direct autocorrelation of soft-x-ray free-electron-laser pulses by timeresolved two-photon double ionization of He. Physical Review A, 80, (2009), 025402

Murphy, J.B. \& Pellegrini, C (1985). Generation of high-intensity coherent radiation in the soft-x-ray and vacuum-ultraviolet region. Journal of the Optical Society of America B, 2, (1985) 259-264

Nisoli, M. \& Sansone, G. (2009). New frontiers in attosecond science. Progress in Quantum Electronics, 33, (2009) 17-59

Oepts, D.; van der Meer, A.F.G.; van Amersfoort, P.W. (1995). The Free-Electron-Laser user facility FELIX. Infrared Physics Technology, 1, (1995) 297-308

Reiche, S. (2006). Transverse coherence properties of the LCLS x-ray beam. Proceedings of FEL, pp. 126-129, Bessy, Berlin, Germany

Saldin, E.L.; Schneidmiller, E.A. \& Yurkov, M.V. (1999). FAST: a three-dimensional timedependent FEL simulation code, Nuclear Instruments and Methods in Physics Research A, 429, (199), 233-237

Saldin, E.L.; Schneidmiller, E.A. \& Yurkov, M.V. (2000a). Optimization of a seeding option for the VUV free electron laser at DESY, Nuclear Instruments and methods in Physics research $A, 445,(2000)$ 178-182

Saldin, E.L.; Schneidmiller, E.A. \& Yurkov, M.V. (2000b). Diffraction effects in the selfamplified spontaneous emission FEL, Optics Communications, 186, (2000) 185-209

Saldin, E.L.; Schneidmiller, E.A. \& Yurkov, M.V. (2001). Limitations of the transverse coherence in the self-amplified spontaneous emission FEL, Nuclear Instruments and Methods in Physics Research A, 475, (2001) 92-96

Saldin, E.L.; Schneidmiller, E.A. \& Yurkov, M.V. (2006). Properties of the third harmonic of the radiation from self-amplified spontaneous emission free electron laser, Physical review special topics - accelerators and beams, 9, (2006) 030702

Salières, P.; L'Huillier, A. \& Lewenstein M. (1995). Coherence Control of High-Order Harmonics, Physical Review Letters, 74, (1995) 3776-3779

Schlotter, W.; Sorgenfrei, F.; Beeck, T.; Beye, M.; Gieschen, S.; Meyer, H.; Nagasono, M.; Föhlisch, A. \& Wurth, W. (2010). Longitudinal coherene measurements of an extreme-ultraviolet free-electron laser, Optics Letters, 35, (2010) 372-374

Sheehy, B.; Clarke, J.A.; Dunning, J.; Thompson N.R. \& McNeil B.W.J. (2006). High Harmonic Seeding and the 4GLS XUV-FEL. Proceedings of FLS, pp. 36-38, Hamburg, Germany 
Singer, A.; Vartanyants, I. A.; Kuhlmann, M.; Duesterer, S.; Treusch, R \& Feldhaus, J. (2008). Transverse-Coherence Properties of the Free-Electron-Laser FLASH at DESY. Physical Review Letters, 101, (2008) 254801

Vartanyants, I.A. \& Singer, A. (2010). Coherence properties of hard x-ray synchrotron sources and x-ray free-electron lasers, New Journal of Physics 12, (2010) 035004

$\mathrm{Yu}$, L.H. (1991). Generation of intense UV radiation by subharmonically seeded single-pass free-electron lasers, Physical Review Letters, 44, (1991) 5178-5193

Yu, L.H.; Babzien, M.; Ben-Zvi, I.; DiMauro, L. F.; Doyuran, A.; Graves, W.; Johnson, E.; Krinsky, S.; Malone, R.; Pogorelsky, I.; Skaritka, J.; Rakowsky, G.; Solomon, L.; Wang, X. J.; Woodle, M.; Yakimenko, V.; Biedron, S. G.; Galayda, J. N.; Gluskin, E.; Jagger, J. ; Sajaev V. \& Vasserman I. (2000). First lasing of a high-gain harmonic generation free-electron laser experiment. Nuclear Instruments and Methods in Physics Research A, 445, (2000) 301-306

Yu, L.H.; DiMauro, L.; Doyuran, A.; Grave, W.S.; Johnson, E.D.; Heese, R.; Krinski, S.; Loos, H.; Murphy, J.B.; Rabowsky, G.; Rose, J.; Shaftan, T.; Sheehy, B.; Skaritka, J.; Wang, X.J. \& Zu, W. (2003). First Ultraviolet High-Gain Harmonic-Generation FreeElectron Laser. Physical Review Letters, 91, (2003) 074801

Zerne, R.; Altucci, C.; Bellini, M.; Gaarde, M. B.; Hänsch, T. W.; L’Huillier, A.; Lyngå, C.; Wahlström, C.-G. (1997). Phase-Locked High-Order Harmonic Sources, Physical Review Letters, 79, (1997) 1006-1009 


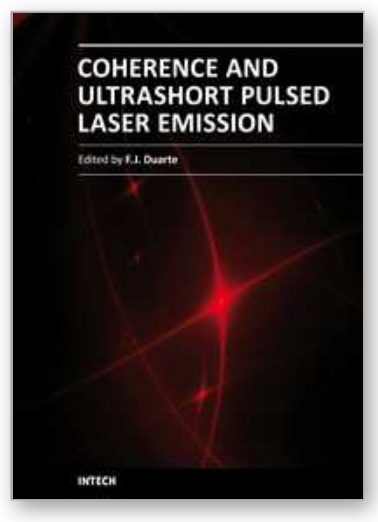

\section{Coherence and Ultrashort Pulse Laser Emission}

Edited by Dr. F. J. Duarte

ISBN 978-953-307-242-5

Hard cover, 688 pages

Publisher InTech

Published online 30, November, 2010

Published in print edition November, 2010

In this volume, recent contributions on coherence provide a useful perspective on the diversity of various coherent sources of emission and coherent related phenomena of current interest. These papers provide a preamble for a larger collection of contributions on ultrashort pulse laser generation and ultrashort pulse laser phenomena. Papers on ultrashort pulse phenomena include works on few cycle pulses, high-power generation, propagation in various media, to various applications of current interest. Undoubtedly, Coherence and Ultrashort Pulse Emission offers a rich and practical perspective on this rapidly evolving field.

\section{How to reference}

In order to correctly reference this scholarly work, feel free to copy and paste the following:

Sebastian Roling and Helmut Zacharias (2010). Coherence of XUV Laser Sources, Coherence and Ultrashort Pulse Laser Emission, Dr. F. J. Duarte (Ed.), ISBN: 978-953-307-242-5, InTech, Available from:

http://www.intechopen.com/books/coherence-and-ultrashort-pulse-laser-emission/coherence-of-xuv-lasersources

\section{INTECH}

open science | open minds

\section{InTech Europe}

University Campus STeP Ri

Slavka Krautzeka 83/A

51000 Rijeka, Croatia

Phone: +385 (51) 770447

Fax: +385 (51) 686166

www.intechopen.com

\section{InTech China}

Unit 405, Office Block, Hotel Equatorial Shanghai

No.65, Yan An Road (West), Shanghai, 200040, China

中国上海市延安西路65号上海国际贵都大饭店办公楼 405 单元

Phone: +86-21-62489820

Fax: +86-21-62489821 
(C) 2010 The Author(s). Licensee IntechOpen. This chapter is distributed under the terms of the Creative Commons Attribution-NonCommercialShareAlike-3.0 License, which permits use, distribution and reproduction for non-commercial purposes, provided the original is properly cited and derivative works building on this content are distributed under the same license. 